\title{
Die außerordentliche Gesetzgebung im Rahmen der Genfer Protokolle Theorie und Praxis des „,außerordentlichen Kabinettsrates“ 1922-1924
}

\author{
Extraordinary legislation within the framework of the Geneva Protocols (Austria 1922-1924)
}

After World War I the Republic of Austria faced a total state bankruptcy. Further loans, guaranteed by several European states, required an intense reform program involving the League of Nations. The realisation of this program by regular parliamentary legislation seemed dubious. Therefore, a special institution was created - the 'Extraordinary Cabinet Council', which constitutional history regards as an institution between legislation and administration. This paper describes how this 'Extraordinary Cabinet Council' was conceived and set up, which aims it should have served and (based on the protocols in the Austrian State Archives) how it actually worked. On the whole, the 'Extraordinary Cabinet Council' points to the distrust in democratic institutions in the interwar period - even in democratic states.

Keywords: administration - Austria, democracy - Extraordinary Cabinet Council -

Extraordinary legislation - Geneva Protocols - state bankruptcy

\section{Der wirtschaftliche Notstand und die Genfer Protokolle}

Die junge Republik Österreich - der „Staat, den keiner wollte" ${ }^{\prime \prime}$ - befand sich am Beginn der 1920er-Jahre infolge von Kriegsschulden, Ernteund Produktionsausfällen sowie der zur Beruhigung der Bevölkerung erforderlich scheinenden Lebensmittelsubventionen in einer wirtschaftlich katastrophalen Situation. Besonders die Währung stand unter enormem Druck, denn während 1919 zur Bedeckung des Haushaltsdefizits noch erfolgreich eine Staatsanleihe ausgegeben worden war, wurde in der Folge nur mehr auf die Produktion "neuen Geldes“ ge-

\footnotetext{
${ }^{1}$ So der Buchtitel von ANDICS, Staat, den keiner wollte. - Für die Unterstützung bei der Einrichtung des Anmerkungsapparats danke ich Frau Stud.Ass. Andreea Panazan.
}

setzt. Der Geldumlauf, der schon bis 1920 von zwölf auf 30 Milliarden Kronen gestiegen war, erreichte Ende 1921 bereits 174 Milliarden und im August 1922 eine Billion Kronen. Die Preise waren zwischen 1914 und 1922 jährlich um etwa $100 \%$ gestiegen, in der Folge nahmen sie monatlich um $50 \%$ zu. Die Lebenshaltungskosten betrugen im Sommer 1922 das 14-tausendfache von 1914. ${ }^{2}$ Die Nationalbank musste dem durch die Ausgabe immer größerer Banknoten Rechnung tragen: So erschien 1922 im Jänner der 5.000 Kronen-Schein sowie ein 50.000 KronenSchein, im August der 100.000 Kronen-Schein, im September schließlich sogar ein 500.000 Kronen-Schein. ${ }^{3}$ Im Dezember 1924 wurde durch

\footnotetext{
2 SANDGRUBER, Ökonomie und Politik 354f.

${ }^{3}$ Vgl. https://www.oenb.at/Ueber-Uns/Geldmuseum/ Sammlungen/Oesterreichische-Banknoten/Kronen-
} 
die Einführung der Schilling-Währung, wobei ein Schilling 10.000 Kronen entsprach, die Inflation gestoppt. ${ }^{4}$ Der gesamtwirtschaftliche Notstand des Staates wird anhand dieser Kennzahlen deutlich greifbar.

Die besondere Gesetzgebung im Notstand bzw. zu dessen Behebung hatte ihre Grundlage in einem komplexen Regelwerk, den Genfer Protokollen. ${ }^{5}$ Dabei handelte es sich um drei völkerrechtliche Verträge zwischen Österreich einerseits und Großbritannien, Frankreich, Italien und der Tschechoslowakei andererseits. Von diesen drei Abkommen hatte eines seinen Schwerpunkt in der Politik, die beiden anderen hatten wirtschaftliche Fragen zum Gegenstand. Protokoll I enthielt die Anerkennung der politischen Unabhängigkeit, der territorialen Integrität und Souveränität Österreichs durch Großbritannien, Frankreich, Italien und die Tschechoslowakei, im Gegenzug die Verpflichtung der Republik Österreich, "gemäß dem Wortlaute des Art. 88 des Vertrages von Saint-Germain, ihre Unabhängigkeit nicht aufzugeben", also eine Wiederholung des in Österreich vor allem als Anschlussverbot verstandenen Unabhängigkeitsgebotes. ${ }^{6}$

Das zweite Genfer Protokoll hatte vor allem die Garantiebedingungen zum Inhalt, unter denen Österreich an neue Geldmittel herankommen konnte: „Die österreichische Regierung wird unter der Garantie, die sich aus dem vorliegen-

Banknoten-der-Oesterreichisch-ungarischen-

Bank.html (26. 1. 2018).

4 SANDGRUBER, Ökonomie und Politik 363.

${ }^{5}$ Genfer Protokolle 1922 BGBl. 842/1922. Dazu BERCHTOLD, Verfassungsgeschichte 350ff; SANDGRUBER, Ökonomie und Politik 361ff; FREUND, Genfer Protokolle; LADNER, Seipel; MERKL, Völkerbundkontrolle. Die Genfer Protokolle waren insbesondere wiederholt Gegenstand akademischer Qualifikationsarbeiten: BLIEBERGER, Währungskrise; KEUC, Haltung; SCHMID, Auseinandersetzungen; THEINER, Kuratel.

${ }^{6}$ Genfer Protokolle 1922 BGBl. 842/1922, Protokoll Nr. I, Erklärung. den Abkommen ergibt, Staatsobligationen im notwendigen Ausmaß ausgeben können, um einen wirklichen Gesamtertrag von höchstens 650 Millionen Goldkronen zu erreichen. ${ }^{\text {"7 }}$ Für diese Garantie hatte zuvor schon das Finanzkomitee des Völkerbundrates verschiedene mögliche Haftungskonstruktionen bzw. Garantiemodelle aufgezeigt: ${ }^{8}$ Eine solidarische Haftung der garantierenden Staaten für eine einzige österreichische Anleihe war jedoch ebenso verworfen worden wie eine besondere Haftung jedes einzelnen garantierenden Staates für jeweils eine besondere Anleihe. Realisiert wurde schließlich das Konzept der Haftung jedes einzelnen garantierenden Staates für eine Quote an einer gesamten österreichischen Anleihe. Von den 650 Millionen Goldkronen sollten 130 Millionen sogleich für die Rückzahlung von Vorschüssen dienen, die Großbritannien, Frankreich, Italien und die Tschechoslowakei Österreich bereits gewährt hatten; für diesen Betrag hafteten nur die vier genannten Staaten. Mit den weiteren 520 Millionen Goldkronen sollte das österreichische Haushaltsdefizit der beiden Folgejahre 1923 und 1924 abgedeckt werden. Dafür hafteten die vier genannten Staaten zu jeweils $20 \%$, für den Rest erhoffte man sich eine Haftungsübernahme durch künftig allenfalls beitretende Staaten. ${ }^{9} \mathrm{Im}$ Ergebnis garantierten Großbritannien, Frankreich, Italien und die Tschechoslowakei somit für $84 \%$ der Gesamtsumme von 650 Millionen Goldkronen..$^{10}$ Es erstaunt nicht, dass die Garantiemächte angesichts dieser gewaltigen Risikoübernahme Kontrollrechte verlangten: Eine Kontrollkommission, die man entfernt mit einem Gläubigerausschuss vergleichen kann, war

\footnotetext{
${ }^{7}$ Genfer Protokolle 1922 BGBl. 842/1922, Protokoll Nr. II, Art. 1.

${ }^{8}$ Siehe dazu FreUnd, Genfer Protokolle 51f.

${ }^{9}$ Vgl. Freund, Genfer Protokolle 52; LADNER, Seipel 151f; Genfer Protokolle 1922 BGBl. 842/1922, Beilagen zum Protokoll Nr. II.

${ }^{10}$ Genfer Protokolle 1922 BGBl. 842/1922, Protokoll Nr. II, Art. 5.
} 
über ein österreichisches Reformprogramm zu informieren und übte Kontroll- und Zustimmungsrechte insbesondere über die Verwendung der den Gläubigern eingeräumten Pfandrechte an Zöllen und Tabakmonopol sowie im Hinblick auf eine Konvertierung der Anleihen aus. ${ }^{11}$ Schließlich bestimmte das Genfer Protokoll II auch die Einsetzung eines Generalkommissärs durch den Völkerbundrat, dessen Aufgaben sich teilweise aus dem Protokoll III ergeben sollten. ${ }^{12}$ Dies ist insofern bemerkenswert, als der Völkerbund selbst an den Genfer Protokollen gar nicht beteiligt war - es handelte sich demnach um eine Konstruktion, die mit einem Vertrag zu Lasten Dritter vergleichbar ist. Die Garantiemächte nahmen diese Bestimmung letztlich nur im Vertrauen auf ihr politisches Gewicht im Völkerbund in das Genfer Protokoll II auf. ${ }^{13}$

Im Genfer Protokoll III schließlich waren vor allem die österreichischen Verpflichtungen enthalten. Österreich musste sich zur Ratifizierung von Protokoll I (Unabhängigkeitsverpflichtung) verpflichten, ${ }^{14}$ ebenso zur umgehenden (innerhalb von vier Wochen zu erfolgenden) Ausarbeitung eines „Reform- und Sanierungsprogramms" entsprechend den "Grundlinien" im Bericht des Finanzkomitees und gemeinsam mit dem Generalkommissär. Das Ziel des Reformund Sanierungsprogramms war es, „Österreich binnen zwei Jahren die Herstellung eines dauernden Gleichgewichtes in seinem Budget zu ermöglichen". ${ }^{15} \mathrm{Zu}$ dessen Verwirklichung musste sich Österreich bereiterklären, einen Gesetzentwurf zu erarbeiten, „der jede Regie-

\footnotetext{
${ }^{11}$ LADNER, Seipel 158; Genfer Protokolle 1922 BGBl. 1922/842, Protokoll Nr. II, Art. 6ff.

${ }^{12}$ Genfer Protokolle 1922 BGBl. 842/1922, Protokoll Nr. II, Art. 4.

${ }^{13}$ Vgl. MERKL, Völkerbundkontrolle 603.

${ }^{14}$ Genfer Protokolle 1922 BGBl. 842/1922, Protokoll Nr. III, Punkt 1.

${ }^{15}$ Genfer Protokolle 1922 BGBl. 842/1922, Protokoll Nr. III, Punkt 2.
}

rung, die während der nächsten zwei Jahre im Amt sein wird, bevollmächtigt, ohne neuerlich an das Parlament herantreten zu müssen, innerhalb der Grenzen dieses Programmes alle Maßnahmen zu ergreifen, die nach ihrer Meinung notwendig sind, um bis zum Ende dieses Zeitabschnittes das budgetäre Gleichgewicht wiederherzustellen". ${ }^{16}$ Österreich musste sich auch mit der Ernennung des Generalkommissärs einverstanden erklären und dessen Funktionen und Kompetenzen anerkennen, insbesondere jene, die aus der Verpfändung der Einnahmen aus Zöllen und dem Tabakmonopol resultierten. ${ }^{17}$

Die drei Genfer Protokolle waren demnach ineinander verzahnt und bildeten ein komplexes Regelwerk; das Gesamtsystem der Genfer Protokolle veranschaulicht Abbildung 1. Diese Konstruktion diente dazu, einerseits Österreichs Liquidität zu erhalten, andererseits damit die Geldgeber abzusichern - dies jedoch, ohne dass Österreich in eine Art Schuldknechtschaft seiner Geldgeber geriet. Doch während Österreichs direkte Abhängigkeit von Privatgläubigern vermieden wurde, etablierte man mit dem vom Völkerbund bestimmten Generalkommissär ein Organ mit geradezu übermächtiger Stellung und zentraler, umfassender Kontrollkompetenz.

Die Genfer Protokolle hatten Konsequenzen auf mehreren Ebenen: Einerseits wurden Österreichs Einnahmen im Wesentlichen auf jene Zahlungen beschränkt, die über den Generalkommissär flossen, andererseits all jene Maßnahmen verunmöglicht, mit denen sich Österreich bis dahin finanziell über Wasser gehalten hatte. Nachdem der Druck neuer Banknoten oder die Ausgabe neuer Anleihen ausgeschlossen war, verfügte Österreich auf der Einnahmenseite über keinerlei Spielraum mehr, woraus

\footnotetext{
${ }^{16}$ Genfer Protokolle 1922 BGBl. 842/1922, Protokoll Nr. III, Punkt 3.

${ }^{17}$ Genfer Protokolle 1922 BGBl. 842/1922, Protokoll Nr. III, Punkt 4.
} 
der Zwang zu ausgabenseitigen Reformen resultierte. ${ }^{18}$

Zum Generalkommissär wurde Alfred Rudolf Zimmermann (1869-1939) ernannt. Er war von 1906 bis 1923 Bürgermeister der Stadt Rotterdam und hatte noch vor Ende seines dortigen Wirkens mit 15. Dezember 1922 die Aufgabe als Generalkommissär des Völkerbundes für Österreich übernommen. Infolge seiner starken, die Souveränität Österreichs massiv einschränkenden Stellung und der an seine Person geknüpften Sparmaßnahmen avancierte Zimmermann als „Ersatzkaiser von Österreich“ zum Feindbild insbesondere der sozialdemokratischen Partei. ${ }^{19}$

\section{Die Umsetzung des Genfer Protokolls III in Österreich: Wiederaufbaugesetz, Vollmachtengesetz und außerordentlicher Kabinettsrat}

\section{A) Überblick}

Der Verpflichtung zur Ausarbeitung eines „Reform- und Sanierungsprogramms" entledigte sich Österreich zunächst durch das Bundesgesetz vom 27. November 1922 , über die zur Aufrichtung der Staats- und Volkswirtschaft der Republik Österreich zu treffenden Maßnahmen", das so genannte Wiederaufbaugesetz, BGB1. 843/1922. ${ }^{20}$ Es enthielt nicht nur eine Reihe von Reformmaßnahmen, sondern bot auch die Grundlage dafür, dass die Bundesregierung „im Rahmen der Grundsätze“ des „Reform- und Finanzprogramms" Verordnungen erlassen konnte. Das Wiederaufbaugesetz erging „in Durchführung der einschlägigen Bestimmungen

\footnotetext{
${ }^{18}$ FREUND, Genfer Protokolle 53ff.

${ }^{19}$ BERGER, Geschichte Österreichs 92.

${ }^{20}$ AdAMOVICH, Grundriss 27; BerchtOld, Verfassungsgeschichte $354 f$.
}

der Genfer Protokolle" und war auf zwei Jahre befristet. $^{21}$

Daneben wurde das Bundesverfassungsgesetz vom 26. November 1922 „über die Ausübung der außerordentlichen Vollmachten, die der Bundesregierung gemäß dem Genfer Protokoll Nummer III vom 4. Oktober 1922 eingeräumt werden", BGBl. 844/1922, erlassen, das bis zum 31. Dezember 1924 befristete sog. „Vollmachtengesetz".22 Dieses lieferte die Grundlage für eine außerordentliche Gesetzgebung in Form "besonderer Regierungsbeschlüsse“ eines sog. „außerordentlichen Kabinettsrates"23 (in der Folge abgekürzt als AoKR).

\section{B) Zum Vollmachtengesetz}

Mit dem Vollmachtengesetz BGBl. 844/1922 verwirklichte Österreich seine Verpflichtung zur Vorlage eines Gesetzentwurfes, „der jede Regierung, die während der nächsten zwei Jahre im Amt sein wird, bevollmächtigt, ohne neuerlich an das Parlament herantreten zu müssen, innerhalb der Grenzen dieses Programms alle Maßnahmen zu ergreifen, die nach ihrer Meinung notwendig sind, um bis zum Ende dieses Zeitabschnittes das budgetäre Gleichgewicht wiederherzustellen". ${ }^{24}$

Das Vollmachtengesetz hatte eine zwar kurze, doch bewegte Gesetzgebungsgeschichte hinter sich. ${ }^{25}$ Dabei wurden Wiederaufbaugesetz und Vollmachtengesetz in der parlamentarischen Diskussion als Einheit verstanden und diskutiert. Die Sozialdemokraten waren zunächst generell gegen die Genfer Protokolle eingestellt gewesen und hatten eine "Selbsthilfeaktion"

\footnotetext{
${ }^{21}$ Wiederaufbaugesetz 1922 BGBl. 843/1922, Abschnitt E, § 1 Abs. 1 und 2.

${ }^{22}$ AdAmOVICH, Grundriss 27f; Berchtold, Verfassungsgeschichte $359 \mathrm{ff}$.

${ }^{23}$ Vollmachtengesetz 1922 BGBl. 844/1922, Art. I.

${ }^{24}$ Genfer Protokolle 1922 BGBl. 842/1922, Protokoll Nr. III, Punkt 3.

${ }^{25}$ ANONYMUS, Seipels neuer Vorschlag 2.
} 
durch Inanspruchnahme von Geld der „Reichen" favorisiert. Die Genfer Protokolle erschienen ihnen als „Verbrechen an der Unabhängigkeit der Republik“, als „Knechtungsvertrag“.26 Der erste Entwurf der Bundesregierung sah eine schlichte parlamentarische Bevollmächtigung der Bundesregierung vor, die damit eine „Finanzdiktatur" errichten hätte können. Besonders empörend erschien der Opposition der Umstand, dass die Regierungsvorlage für diese doch sehr weit gehende Bevollmächtigung der Bundesregierung nicht einmal eine Verfassungsbestimmung vorgesehen hatte. ${ }^{27}$ Nachdem sich für eine derartigen Entwurf keine Mehrheit gefunden hätte, bot Bundeskanzler Ignaz Seipel als Kompromiss zunächst eine nachträgliche parlamentarische Kontrolle der von der Bundesregierung auf Grundlage der Vollmacht gesetzten Maßnahmen an. Dies wäre jedoch nicht zielführend gewesen, weil damit jede Verordnung der Bundesregierung von einer solchen nachträglichen Prüfung und letztlich von der Aufhebung durch den Verfassungsgerichtshof bedroht erschien. ${ }^{28}$ In einem neuen Angebot schlug Seipel schließlich eine verfassungsgesetzliche Regelung vor, wonach ein vom Nationalrat nach Verhältniswahlrecht gewählter Staatsrat die Bundesregierung bei der Ausübung ihrer Vollmacht kontrollieren sollte. Allerdings hätte ein solcher Staatsrat nicht dem Genfer Protokoll III entsprochen, das ausdrücklich eine Bevollmächtigung der „Regierung“ verlangt hatte. Im Hinblick darauf wurde schließlich eine Verstärkung der Regierung durch gewählte Staatsräte in einem „Kabinettsrat" konzipiert. ${ }^{29}$ Fast schon be-

\footnotetext{
${ }^{26}$ BERCHTOLD, Verfassungsgeschichte 351ff, 353. Vgl. allgemein dazu KeUC, Haltung.

${ }^{27}$ BERCHTOLD, Verfassungsgeschichte 355.

${ }^{28}$ Ebd.; StenProtNR I. GP, 145. Sitzung v. 6. 11. 1922, 4553.

${ }^{29}$ BERCHTOLD, Verfassungsgeschichte 355f; BERGER, Geschichte Österreichs 91f. Durch die „Schaffung eines eigenen Verfassungsgesetzes" hatte sich in der medialen Wahrnehmung „die parlamentarische Lage
}

wundernd schrieb die Arbeiterzeitung: „In der Tat bietet Herr Seipel den Völkerbundsdelegierten die Form, daß die Körperschaft, die über die finanziellen Maßregeln entscheidet, Kabinettsrat heißt, also als eine bloße Verstärkung der Regierung hingestellt wird; den Sozialdemokraten aber die Sache, daß die finanziellen Maßregeln nicht verfügt werden dürfen ohne vorherige Genehmigung einer parlamentarischen Körperschaft. Man sieht: eine der gefährlichsten Bestimmungen des Genfer Vertrages ist schon ganz hübsch durchlöchert. "“30 Zugleich kamen die Christlichsozialen den Wünschen der Opposition auch durch eine Änderung der Geschäftsordnung des Nationalrates entgegen; dieser sollte künftig schon auf Verlangen eines Viertels seiner Mitglieder einberufen werden, wofür also die Stimmen der Sozialdemokraten genügten. Die entsprechenden Beschlüsse sollten bis 27. November 1922 fallen. $^{31}$

Zwar konnten es die Sozialdemokraten bei dem sich abzeichnenden Kompromiss als Erfolg für sich verbuchen, eine Finanzdiktatur der Regierung vereitelt zu haben, doch wollten sie nicht den Eindruck mangelnden Widerstandsgeistes erwecken. Die Öffentlichkeit sollte sehen, dass sie mit aller Kraft gegen die Übertragung von Gesetzgebungsbefugnissen an die Regierung kämpfen würden; daher erhoben sie im Bundesrat Einspruch gegen den Nationalratsbeschluss. Zwar hatten die Regierungsparteien auch hier eine knappe Mehrheit (Sozialdemokraten 24, Christlichsoziale 23, Großdeutsche 2), praktisch bestand jedoch eine Pattstellung, weil ein Christlichsozialer als Vorsitzender kein Stimmrecht

bedeutend entspannt “: Salzburger Chronik, Nr. 254 v. 19./20. 11. 1922, 6 .

${ }^{30}$ Arbeiter-Zeitung, Nr. 310 v. 18. 11.1922, 2.

${ }^{31}$ BERCHTOLD, Verfassungsgeschichte 355f. Diese erleichterte Einberufung des Nationalrates wurde noch im Dezember 1922 genutzt, als die Sozialdemokraten Steuererhöhungen für Getränke und Zucker diskutieren wollten: vgl. Neues Wiener Tagblatt Nr. 345 v. 27. 12. 1922, 4. 
hatte. Daher drohte eine Situation, in welcher im Bundesrat gar kein Beschluss zustande kommen konnte; in diesem Fall wäre das Gesetz mangels Einspruchs des Bundesrates zwar in Kraft getreten, dies aber erst nach acht Wochen - im Hinblick auf Finanzkrise und Genfer Protokolle zu spät. Nachdem also die Zeit drängte, wurde mittels einer Parteienabrede ein Ausweg konzertiert: Die Bundesräte der Regierungsparteien verließen vor der Abstimmung den Saal, sodass der Bundesrat mit den Stimmen der verbliebenen Sozialdemokraten Einspruch gegen das Wiederaufbaugesetz ${ }^{32}$ erheben konnte, womit diese politisch ihr Gesicht gewahrt, zugleich aber einen raschen Beharrungsbeschluss des Nationalrates ermöglicht hatten. Dieser Beharrungsbeschluss wurde schon am 3. Dezember gefasst und machte den Weg frei auch zum Vollmachtengesetz. ${ }^{33}$

Ungeachtet aller Überlegungen im Vorfeld entsprach das Vollmachtengesetz letztlich nicht dem Genfer Protokoll III. Nach dessen Vorstellungen sollte ja ,jede Regierung“ bevollmächtigt werden, die „,während der nächsten zwei Jahre im Amt sein wird, [...] ohne neuerlich an das

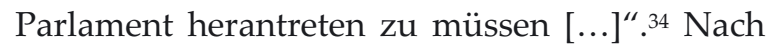
dem Vollmachtengesetz wurde hingegen zur „Ausübung der außerordentlichen Vollmachten [...] der Bundesregierung[!]“ ein „außerordentlicher Kabinettsrat" eingesetzt. ${ }^{35}$ Es erscheint als ein raffiniertes begriffliches Täuschungsmanöver, zur Ausübung von Vollmachten des Organs A ein Organ Non-A vorzusehen. Diese Widersprüchlichkeit ist nicht bloß redaktionelles Versehen, sondern hatte offensichtlich System: Zwar wurden „der Bundesregierung [...] fol-

\footnotetext{
${ }^{32}$ Der Einspruch richtete sich nicht auch gegen das hier vor allem interessierende Vollmachtengesetz.

${ }^{33}$ BERCHTOLD, Verfassungsgeschichte 358f; Sten-

ProtBR I. GP, 48. Sitzung v. 29. 11. 1922, 744.

${ }^{34}$ Genfer Protokolle 1922 BGBl. 842/1922, Protokoll

Nr. 3, Punkt 3.

${ }^{35}$ Vollmachtengesetz 1922 BGBl. 844/1922, Art. I.
}

gende Vollmachten erteilt", Beschlüsse „gemäß Abs. 1“ jedoch "als besondere Regierungsbeschlüsse durch den außerordentlichen Kabinettsrat gefasst". ${ }^{36}$

Wiederaufbaugesetz und Vollmachtengesetz standen nicht nur gesetzgebungsgeschichtlich in Zusammenhang, sondern auch strukturell. Besondere Regierungsbeschlüsse konnten nämlich in verschiedenen Zusammenhängen gefasst werden: Einerseits waren sie notwendig, wenn zum Reform- und Finanzprogramm des Wiederaufbaugesetzes außerhalb des Rahmens der von der Bundesregierung ohnehin regulär zu erlassenden Durchführungsverordnungen weitere Verordnungen zu treffen waren; andererseits waren notwendige Ergänzungen und Abänderungen des Reform- und Finanzprogramms samt dazu gehörigen Durchführungsverordnungen ausschließlich auf der Grundlage des Vollmachtengesetzes möglich. ${ }^{37}$ Die gemeinsame Grenze sowohl für weitere Verordnungen zum Wiederaufbaugesetz als auch für Verordnungen auf Grundlage des Vollmachtengesetzes bildete die bestehende Verfassung: Sie durfte weder durch Verordnungen der Bundesregierung gemäß Wiederaufbaugesetz noch durch Maßnahmen des AoKR aufgrund des Vollmachtengesetzes abgeändert werden. Natürlich gab es auch Abgrenzungsprobleme zwischen Verordnungen der Bundesregierung im Rahmen des Wiederaufbaugesetzes und solchen außerhalb desselben unter Befassung des AoKR;38 über solche Abgrenzungsprobleme hatte letztlich der Verfassungsgerichtshof zu entscheiden. ${ }^{39}$

\footnotetext{
36 Vollmachtengesetz 1922 BGBl. 844/1922, Art. III.

${ }^{37}$ Vollmachtengesetz 1922 BGBl. 844/1922, Art. III.

38 WITTMAYER, Staatsrecht 389.

${ }^{39}$ BERCHTOLD, Verfassungsgeschichte 361; vgl. z.B. VfSlg 176/1923.
} 


\section{C) Der außerordentliche Kabinettsrat - Zusammensetzung und Verfahren}

Dem Vollmachtengesetz gemäß bestand der AoKR aus der Bundesregierung sowie aus 26 vom Nationalrat nach Verhältniswahlrecht (Listen) gewählten Mitgliedern. ${ }^{40}$ Wählbar waren nur Mitglieder des Nationalrates und des Bundesrates; es handelte sich um ein unbesoldetes Ehrenamt, das unabhängig von der Gesetzgebungsperiode des Nationalrates und bis zu einer Neuwahl auszuüben war. ${ }^{41}$

Die erste Wahl fand am 7. Dezember 1922 statt, wobei von den $26 \mathrm{zu}$ wählenden Staatsräten 12 auf die Christlichsozialen, 10 auf die Sozialdemokraten, 3 auf die Großdeutschen und 1 auf die Bauernpartei entfielen. ${ }^{42}$ Dieses Verhältnis verschob sich in der zweiten Gesetzgebungsperiode den geänderten Kräfteverhältnissen der Parteien folgend leicht; am 24. November 1923 wurden 13 Christlichsoziale, 11 Sozialdemokraten und 2 Staatsräte für die Großdeutsche Volkspartei und den Verband des Landbundes gewählt. ${ }^{43}$ Bei beiden Wahlen wurde zwar zwischen Hauptmitgliedern und Ersatzmitgliedern unterschieden, in der Folge gingen die Parteien mit dem Wahlergebnis jedoch flexibel um, so-

\footnotetext{
${ }^{40}$ Vollmachtengesetz 1922 BGBl. 844/1922, Art. I. Die Zahl wurde letztlich, sozialdemokratischen Forderungen folgend, so festgesetzt, damit alle Klubs im AoKR vertreten waren; damit erschienen die Staatsräte als „Nationalrat im Kleinen“: BERCHTOLD, Verfassungsgeschichte 360 . Noch von 25 Staatsräten schreibt etwa die Arbeiter-Zeitung, Nr. 310 v. 18.11.1922, 2, von hier übernommen in andere sozialdemokratische Zeitungen, etwa Salzburger Wacht, Nr. 260 v. 20.11.1922, 1; ebenso noch die kommunistische Rote Fahne, Nr. 1072 v. 19. 11. 1922, 1.

${ }^{41}$ Vollmachtengesetz 1922 BGBl. 844/1922, Art. II Abs. 1. Zunächst war man davon ausgegangen, dass die Mitgliedschaft im AoKR nicht auf Parlamentarier beschränkt sein würde, vgl. Neues Wiener Tagblatt, Nr. 307 v. 17. 11. 1922.

${ }^{42}$ StenProtNR I. GP, 157. Sitzung v. 7. 12. 1922, $5013 f$. BERCHTOLD, Verfassungsgeschichte 360f.

${ }^{43}$ StenProtNR II. GP, 4. Sitzung v. 24. 11. 1923, 77.
}

dass manche Hauptmitglieder seltener im AoKR erschienen als manche Ersatzmitglieder. Selbst besonders rege Teilnahme von Ersatzmitgliedern führte nicht zwangsläufig zu einer Aufwertung zum Hauptmitglied; der Christlichsoziale Mathias Partik etwa wurde auch 1923 nur zum Ersatzmitglied gewählt, obwohl er von seiner Partei nach Jodok Fink am öftesten im AoKR gewesen war. Dafür kamen andere, selbst prominente Ersatzmitglieder niemals zum Zug Beispiele dafür sind Friedrich Adler, Stephan Falser oder Sepp Straffner. Bei einer Auswertung der Protokolle ${ }^{44}$ zeigt sich in der Praxis des AoKR eine erhebliche Teilnehmerfluktuation: ${ }^{45}$ Insgesamt traten 69 Personen als Staatsräte auf, von denen immerhin 10 nur ein einziges Mal am AoKR teilnahmen. Trotz - oder wegen - der relativen Beliebigkeit wurden die personellen Wechsel in den Protokollen des AoKR nahezu feierlich als "Mandatsniederlegungen“ deklariert: „Der Vorsitzende bringt dem außerordentlichen Kabinettsrat ein an den Präsidenten des Nationalrates gerichtetes Schreiben der christlichsozialen Vereinigung zur Kenntnis, demzufolge die Abgeordneten Stöckler und Partik sowie die Bundesräte Dr. Rintelen und Zwetzbacher ihre Mandate als Mitglieder des außerordentlichen Kabinettsrates niederlegen und unter die Ersatzmänner treten. An ihrer Stelle werden die Abgeordneten Kollmann, Paulitsch, Rudel-Zeynek und Dr. Weiß in den auBerordentlichen Kabinettsrat entsendet." ${ }^{46}$ Sogleich in der folgenden Sitzung hieß es: „Der Vorsitzende teilt vor Eingang in die Tagesord-

\footnotetext{
${ }^{44}$ Sie erliegen im ÖStA, AdR. Für wertvolle Hilfe bei der Auszählung danke ich Frau StudAss Katharina Planner.

${ }^{45}$ Ein Zusammenhang zwischen dieser Fluktuation und dem Fehlen einer „offiziellen“ Liste der Staatsräte im Rahmen der Indices zu den Stenographischen Protokollen des Nationalrates ist nicht undenkbar. ${ }^{46}$ AoKR Protokoll 2, 2. Diese Terminologie findet sich auch in Zeitungsberichten; vgl. z.B. Wiener Zeitung, Nr. 22 v. 27. 1. 1923, 1
} 
nung mit, daß an Stelle des St.R. Eldersch der Abgeordnete Sever und an Stelle der Staatsräte Hauser und Paulitsch die Abgeordneten Kletzmayr und Partik als Mitglieder in den außerordentlichen Kabinettsrat eintreten." ${ }^{47}$ Paulitsch hatte also nur an einer Sitzung teilgenommen, Partik nur für eine Sitzung gefehlt. Aufgrund der reichlich vorhandenen Ersatzmitglieder wurde das zur Beschlussfassung erforderliche Präsenzquorum (13 von 26 ) stets mühelos erreicht; nur zweimal nahmen weniger als 20 Staatsräte am AoKR teil. Bei allen Sitzungen anwesend war lediglich der sozialdemokratische Abgeordnete Wilhelm Schiegl, der auch als einer der Schriftführer fungierte.48 Zur zweiten Sitzung waren versehentlich sogar 27 Staatsräte erschienen! (Details siehe Tabellen 1 und 2 im Anhang).

Die Sitzungen des AoKR wurden durch den Bundeskanzler einberufen, Beschlüsse wurden bei Anwesenheit von mindestens der Hälfte der Staatsräte gefasst, und zwar in einer Frist von acht Tagen. Diese Frist konnte eventuell auf drei Tage verkürzt werden. Innerhalb dieser Zeit musste die Gelegenheit zu Beratungen in mindestens acht Sitzungen (eventuell nur drei Sitzungen) bestehen..$^{49}$ Der Beschluss erfolgte durch Stimmenmehrheit der Staatsräte, das Ergebnis war ein sogenannter ",besonderer Regierungsbeschluss", wobei insbesondere der Wortbestandteil „Regierung“ der Optik im Hinblick auf die Anforderungen des Genfer Protokolls III diente. ${ }^{50}$ Die Mitglieder der Bundesregierung, obschon Mitglieder des AoKR, stimmten jedoch nicht mit. ${ }^{51}$ Fiel keine Entscheidung des AoKR,

\footnotetext{
${ }^{47}$ AoKR Protokoll 3, 2.

48 AoKR Protokoll 1.

${ }^{49}$ Vollmachtengesetz 1922 BGBl. 844/1922, Art. VI und VII.

${ }^{50}$ ANONYMUS, Gesetz 2; WITTMAYER, Staatsrecht 389.

${ }^{51}$ Beschlüsse kamen zustande, „wenn den bezüglichen Entwürfen der Bundesregierung die Staatsräte (!) durch einen Beschluß zustimmen“: Vollmachten-
}

so konnte die Bundesregierung allein entscheiden. Kundgemacht wurden solche Verordnungen als „Verordnung der Bundesregierung “52 (!) unter Verweis auf BGBl. 844/1922 und den besonderen Regierungsbeschluss des AoKR (vgl. Abbildung 2).

Der AoKR gab sich in seiner ersten Sitzung eine Geschäftsordnung, die im Wesentlichen auf einer Parteienabrede beruhte. Leider ist diese Geschäftsordnung in den Akten des AoKR nicht mehr erhalten; aus den Protokollen ergeben sich jedoch einzelne Bestimmungen sowie insbesondere die subsidiäre analoge Anwendung der Geschäftsordnung des Nationalrates. ${ }^{53} \mathrm{Zu}$ Beginn der Verhandlungen war jeweils ein Berichterstatter zu bestellen. ${ }^{54}$ Schon in der ersten Sitzung des AoKR wurde auf Seipels Vorschlag beschlossen, für die Protokollierung der Beratungen Beamte des Bundeskanzleramts zu verwenden, deren Niederschriften in der Folge vom Vorsitzenden und den Schriftführern zu beglaubigen waren.55 Daneben wurden auch in der "Wiener Zeitung" und anderen Tageszeitungen „Protokolle“ oder protokollartige Berichte publiziert, die sich jedoch von den offiziellen Niederschriften deutlich unterschieden. ${ }^{56}$

Betrachtet man die Verfassungswirklichkeit, so zeigt sich, dass den Schwierigkeiten bei der

gesetz 1922 BGBl. 844/1922, Art. VI; BERCHTOLD, Verfassungsgeschichte 360 .

52 BERCHTOLD, Verfassungsgeschichte 360.

${ }^{53} \mathrm{Vgl}$. etwa eine Kontroverse zwischen den Staatsräten Weiskirchner und Seitz zur Anwendung des § 34 der Geschäftsordnung des Nationalrates: AoKR Protokoll 5, 3 .

${ }^{54} \S 6$ der Geschäftsordnung: AoKR Protokoll 2, 6. Es war allerdings strittig, ob die Wahl des Berichterstatters am Beginn der Sitzung oder am Beginn der Verhandlung erfolgen sollte: AoKR Protokoll 2, 3ff.

${ }^{55}$ Diese Protokolle liegen im AdR vor; eine Edition ist in Planung.

${ }^{56}$ Siehe dazu unten nach Anm. 65. Einen „Sitzungsbericht" liefert z.B. Neues Wiener Tagblatt, Nr. 43 v. 14. 2. 1923, 6. Zur medialen Wahrnehmung der Genfer Protokolle im allgemeinen THEINER, Kuratel. 
dogmatischen Konstruktion der besonderen Regierungsbeschlüsse keine adäquate praktische Bedeutung entsprach. Insgesamt versammelte sich der AoKR zwischen 20. Dezember 1922 und 24. Oktober 1924 nur zu 37 Sitzungen, die durchschnittlich knapp unter zwei Stunden, insgesamt rund 70 Stunden dauerten. ${ }^{57}$ Sie verteilten sich über den Zeitraum seines Bestehens ziemlich ungleich; 31 der 37 Sitzungen fanden in der ersten Jahreshälfte 1923 statt, nur zwei in der zweiten, eine schon 1922, drei noch 1924 (vgl. Tabelle 3). Die Notwendigkeit zur Beratung in mindestens acht Sitzungen führte dazu, dass die Regierung anfangs versuchte, mehr Themen auf die Tagesordnung zu setzen als im Rahmen der entsprechenden Sitzungen abgearbeitet werden konnten. Vizekanzler Dr. Frank bemerkte dazu in der 2. Sitzung, ",daß alle Gegenstände auf der Tagesordnung der heutigen Sitzung stehen und der Lauf der 8-tägigen Frist sowie die Zählung der 8 Sitzungen nicht davon berührt werde, ob ein Gegenstand in der betreffenden Sitzung verhandelt wurde oder nicht".58 Auch in der Folge kam dem Vorsitzenden besondere Bedeutung für die Bestimmung der achttägigen Frist zu: „Der Vorsitzende bestimmt den heutigen Tag als ersten Beratungstag für diese Gegenstände" ${ }^{\prime 59}$ - und setzte damit den Fristenlauf in Gang. Im Sinne der Fristwahrung wurde auch einmal an einem Sonntag verhandelt - allerdings wurde dies von den Sozialdemokraten, die an diesem Tag ihren niederösterreichischen Landesparteitag abhielten, vor allem als Provo-

\footnotetext{
${ }^{57}$ Dazu wurden die Angaben der Protokolle in Einheiten zu je 1/4 Stunde umgerechnet; die Anzahl dieser Einheiten lag zwischen 1 (8. Sitzung, lediglich zur Verlängerung der achttägigen Frist) und 15 (3. Sitzung), also zwischen 15 Minuten und 3 Stunden 45 Minuten. Vgl. dazu Abbildung 3. Der Sitzungsort war in den Protokollen nicht vermerkt; von der Wiener Zeitung ist als solcher der Budgetsaal des Nationalrates überliefert: Wiener Zeitung, Nr. 15 v. 19.1. 1923, 3.

58 AoKR Protokoll 2, 5

${ }^{59}$ AoKR Protokoll 32, 2.
}

kation und "herausfordernde Illoyalität" empfunden. ${ }^{60}$ Nur einmal wurde die Frist auf drei Tage verkürzt. ${ }^{61}$

Im Zusammenhang mit dem Angestelltenabbaugesetz kam es nicht zu einem rechtzeitigen Beschluss, sodass die entsprechende Verordnung schließlich allein von der Bundesregierung erlassen werden konnte. Dies hatte eine bemerkenswerte Vorgeschichte. Nach mehreren Sitzungen zur Generaldebatte und eigens erfolgter Verlängerung der 8-tägigen Frist sollte am 6. Februar 1923 die Spezialdebatte beginnen. Vizekanzler Dr. Frank wollte diese Spezialdebatte über alle Paragraphen gemeinsam durchführen, was nach Ansicht der Sozialdemokraten eben gerade nicht Zweck einer Spezialdebatte war. Daran entzündete sich ein heftiger Streit, der schließlich in Schreiduellen und Handgreiflichkeiten endete. Anhand dieses Ereignisses werden die Unterschiede zwischen den offiziellen Protokollen und den in den Tageszeitungen lancierten „Protokollen“ gut erkennbar.62 Die offizielle Niederschrift vermerkt: „Die Abstimmung vollzieht sich unter heftigen Protestrufen der der sozialdemokratischen Partei angehörenden Staatsräte und ergibt die Annahme des Vorschlages des Vorsitzenden mit Stimmenmehrheit. Der Vorsitzende erklärt, in diesem Sinne vorgehen und zunächst mehrere ihm zugekommene Abänderungsanträge zur Verlesung bringen zu wollen. Diese Erklärung führt zu Lärmszenen und St.R. Schiegl schleudert eine Aschenschale in der Richtung des Vizekanzlers Dr. Frank auf den Tisch. Der Vorsitzende ruft St.R. Schiegl wegen dieses Verhaltens zur Ordnung. St.R. Seitz übt an dem Vorsitzenden wegen Erteilung dieses Ordnungsrufes Kritik und

\footnotetext{
${ }^{60}$ AoKR Protokoll 4, 7.

${ }^{61}$ AoKR Protokoll 14, 2.

${ }^{62} \mathrm{Vgl}$. neben den in der Folge zitierten "Protokollen“ u.a. Berichte und Kommentare in: Reichspost, Nr. 36 v. 7. 2. 1923; Neue Freie Presse, Nr. 20981 v. 7. 2. 1923, 3; Arbeiterzeitung, Nr. 36 v. 7. 2. 1923, 2.
} 
wird wegen der hiebei gebrauchten Worte gleichfalls zur Ordnung gerufen. Da unter fortdauernden heftigen Auseinandersetzungen zwischen den Angehörigen der Mehrheitsparteien und der Minderheitspartei St.R. Seitz dem Vorsitzenden die der Abstimmung zugrundeliegende Vorlage entreisst und vernichtet, schliesst dieser um 1/2 6 Uhr nachmittags die Sitzung." 63 In der Wiener Zeitung war zu lesen: „Bei der Abstimmung wird der Vorschlag des Vorsitzenden unter lärmenden Zwischenrufen der Sozialdemokraten mit Mehrheit angenommen. Abg. Seitz: Das ist ganz ungültig und absolut rechtswidrig! (Großer Lärm) Vizekanzler Dr. Frank will nunmehr zur Verlesung der zur Spezialdebatte vorliegenden Anträge schreiten, wird jedoch durch stürmische und lärmende Protestrufe der Sozialdemokraten daran gehindert. Nachdem der Lärm und die stürmischen Protestrufe der Sozialdemokraten längere Zeit angedauert haben und es zu sehr heftigen Auseinandersetzungen zwischen den Abgeordneten der Mehrheit und der Minderheit gekommen war, schließt der Vorsitzende um $1 / 2 \quad 6$ Uhr nachmittags die Sitzung." 64 Die Leser der Illustrierten Kronen-Zeitung wurden unter dem Titel „Ein Handgemenge im Kabinettsrat. Präsident Seitz gegen Vizekanzler Frank. Zündsteine und Tintenfässer als Wurfgeschosse [...]“ noch plastischer in die Sitzung geführt: „Vors. Dr. Frank: Ich wüßte nicht, was da ungesetzlich wäre. Seitz (mit der Faust auf den Tisch schlagend): Sie sind ein junger Mann ${ }^{65}$ und kein Vorsitzender! Schweigen Sie! Ich dulde keine Ungesetzlichkeit! Dr. Frank: Aber beruhigen Sie sich doch, ich verstehe Ihre Aufregung nicht. Seitz (hochrot im Gesicht): Wenn Sie das Gesetz mit Füßen treten, lassen wir sie nicht verhandeln. Der Seipel soll

\footnotetext{
${ }^{63}$ AoKR Protokoll 11 (unpag.)

${ }^{64}$ Wiener Zeitung, Nr. 30 v. 7. 2. 1923, 1.

${ }^{65}$ Frank war 1876 geboren, also 47 Jahre alt; Seitz, 1869 geboren, nur 7 Jahre älter: vgl.

https://www.parlament.gv.at/WWER/PARL/.
}

her und den Vorsitz führen. Die Aufregung des Abg. Seitz greift nun auf seine Parteigenossen über, die bisher ganz ruhig gewesen sind. Schiegl (Soz.) ergreift einen schweren irdenen Zündstein, schleudert ihn gegen den Vizekanzler Frank und trifft diesen am Arm. [...] Als der Lärm sich etwas gelegt hatte, erteilte [Frank] dem Abg. Seitz den Ordnungsruf. Seitz: Das ist das höchste! Ein grüner Junge ruft mich zur Ordnung! Dr. Schönbauer: Eine schöne Gesellschaft! Zelenka: Wir verbieten uns diese Beleidigung. Ihr Gesindel! Seitz springt unerwartet auf und stürzt sich auf Vizekanzler Dr. Frank, reißt ihm einen Papierbogen aus der Hand, faßt dann den Vizekanzler an der Brust und schüttelt ihn. Vizekanzler Dr. Frank schüttelt den Angreifer kräftig ab. [...]"66 Auch wenn die Arbeiterzeitung von einer „unverschämten Lüge“ schrieb und "einen gewalttätigen Angriff“ als konstruiert darstellte, um den Eindruck einer moralischen Niederlage abzuschwächen, ${ }^{67}$ so hatten die Sozialdemokraten jedenfalls eine politische Niederlage erlitten, denn, wie die Wiener Zeitung auch sogleich ihre Leser informierte, hatten die "Ereignisse" im AoKR "die Abstimmung über die dort eingebrachten Anträge verhindert". ${ }^{68}$ Damit war die vom Vollmachtengesetz vorgesehene Frist ergebnislos abgelaufen, sodass die entsprechende Verordnung von der Bundesregierung allein erlassen werden konnte. ${ }^{69}$ Angesichts dieses Eklats verwundert es ein wenig, dass zur Verfahrensbeschleunigung später wiederholt General- und Spezialdebatte zusammengezogen wurden, ohne dass daran Kritik geübt wurde. ${ }^{70}$

Die Zahl der besonderen Regierungsbeschlüsse des AoKR blieb überschaubar: Insgesamt konnten bei einer Durchsicht des Bundesgesetzblattes

\footnotetext{
${ }^{66}$ Illustrierte Kronen-Zeitung, Nr. 8281 v. 7. 2. 1923, 5.

${ }^{67}$ Arbeiterzeitung, Nr. 36 v. 7. 2. 1923, 2.

68 Wiener Zeitung, Nr. 30 v. 7. 2. 1923, 1.

${ }^{69}$ Vollmachtengesetz 1922 BGBl. 844/1922, Art. VII.

${ }^{70} \mathrm{Vgl}$. AoKR Protokolle 30 bis 34 .
} 
nur 19 einschlägige Verordnungen nachgewiesen werden, die sich auf einen Zeitraum zwischen dem ersten und dritten Quartal 1923 konzentrieren (vgl. Abbildung 4). ${ }^{71}$ Die durch besondere Regierungsbeschlüsse geregelten Fragen gehörten zwar nicht zu den Kernbereichen der Rechtsordnung, deren Veränderungen wissenschaftlichem Interesse begegnen, sie waren jedoch durchaus vielfältig; geregelt wurden Pensionsbeiträge der Bundesangestellten, Auflassung der Berghauptmannschaften, Besoldungsgesetz, Bundesangestelltenabbau, Umsatzsteuer, Wehrgesetz, Kollegiengelder, Zölle, oberste Bundesverwaltung, Vereinigung des Telegrafen-, Fernsprech-und Postwesens, Personalsteuer, Erwerbssteuer, Verwaltungsverfahrensrecht, Verfahren zum gewerblichen Rechtsschutz, Eichwesen, Gewerbeförderungswesen (vgl. im Detail Tabelle 4). Diesen unterschiedlichen Materien entsprach ein unterschiedliches Interesse an den Sitzungen des AoKR. Die "Wichtigkeit" der verschiedenen Sitzungen ergibt sich aus verschiedenen Indikatoren wie dem Umfang der Sitzungsprotokolle, der Dauer der Sitzungen oder dem Ausmaß der Teilnahme von Regierungsmitgliedern (vgl. Abbildung 3). ${ }^{72}$ Anhand dieser Parameter lässt sich etwa erkennen, dass die Neuordnung der obersten Bundesverwaltung für eher wichtig, die Auflassung der Normal-Eichungskommission für eher unwichtig angesehen wurde. ${ }^{73}$

Der Blick auf die Verfassungswirklichkeit zeigt auch, dass die "Regierung“ als solche bei der Fassung "besonderer Regierungsbeschlüsse" im AoKR keine bedeutende Rolle spielte. Dies lag

\footnotetext{
${ }^{71}$ BERCHTOLD, Verfassungsgeschichte 361, nennt 18 (offenbar ohne BGBl. 409/1924). Vgl. im Detail unten Tabelle 4. Für die Durchsicht des BGBl. danke ich meiner Mitarbeiterin Mag. Marie-Therese Mundsperger.

${ }^{72}$ Hier ist z. B. zu erkennen, dass der oben beschriebene Seitz-Frank-Eklat in der darauffolgenden Sitzung zu einem Anstieg aller drei Parameter führte.

73 27. bzw. 34. Sitzung.
}

wohl nicht nur am fehlenden Stimmrecht. Abgesehen von einzelnen Höhepunkten wie den ersten Sitzungen des AoKR oder jenen Sitzungen, bei denen es um die Organe der obersten Bundesverwaltung ging, blieb auch die Beteiligung von Ministern in der Regel gering: An den 37 Sitzungen des AoKR nahmen zwar bis zu 8 Regierungsmitglieder teil, durchschnittlich waren es jedoch bloß drei (vgl. Abbildung 3). Stets war zumindest ein Regierungsmitglied anwesend, das den Vorsitz führte (neben Bundeskanzler Seipel insbesondere Vizekanzler Frank, siehe oben). Die Regierung musste auch gar nicht anwesend sein, denn sie konnte sich ohnehin auf ihre parlamentarische Mehrheit verlassen, die auch den Staatsrat dominierte, Berichterstatter wählte und die sozialdemokratische Opposition niederstimmte. Diese konnte daher kaum etwas bewirken. ${ }^{74}$ Insofern entspricht die juristisch fragwürdige Terminologie der „besonderen Regierungsbeschlüsse" durchaus der politischen Realität, denn die Beschlüsse wurden von der Regierungsmehrheit gefasst.

\section{Abschließende Überlegungen: Verkleinertes Parlament oder erweiterte Regierung?}

Ungeachtet der geringen praktischen Bedeutung stellt sich die dogmatische Frage nach der Einordnung der besonderen Regierungsbeschlüsse im Spannungsfeld von Legislative und Exekuti-

\footnotetext{
${ }^{74}$ Geradezu sensationell mutet auf den ersten Blick die Annahme eines Antrags von Otto Bauer in der 32. Sitzung an - er hatte aber lediglich den Abbruch der Sitzung beantragt. Einstimmig angenommen wurde nur ein universitätspolitischer Resolutionsantrag von Schönbauer in der 18. Sitzung; vgl. dazu Wiener Zeitung, Nr. 62 v. 16.3. 1923, 7. Angesichts der engen Fristsetzungen im AoKR übertrieben erscheint die Annahme, die Sozialdemokraten hätten immerhin „Obstruktion" treiben können; vgl. TagesPost, Nr. 81 v. 10. 4. 1923, 2.
} 
ve, also insbesondere die Frage, ob es sich beim AoKR um eine aus ökonomischen Gründen verkleinerte Variante des Parlaments oder um eine erweiterte Regierung handelte. Aus der Sicht der Genfer Protokolle sollte die Gesetzgebung durch die Exekutive erfolgen; wenn man diesen Gedanken weiterführt, handelte es sich also um eine erweiterte Regierung. In diesem Sinne charakterisierte die Reichspost den AoKR als "26gliedrige Regierungskörperschaft" ${ }^{75}$ Aus der Sicht der Opposition, die den Parlamentarismus zu verteidigen bestrebt war, erschien der AoKR - bei Würdigung der Gesetzgebungsgeschichte - eher als ein verkleinertes Parlament, als „Nationalrat im Kleinen",76 mit dem eine Regierungsdiktatur eben gerade verhindert worden war. In diesem Sinne war in der Arbeiterzeitung eine Art alternativer Textierung des Vollmachtengesetzes zu lesen, die dessen „Inhalt" besser gerecht würde: „Die Gesetzgebung in Hinsicht der zur Erzielung des budgetären Gleichgewichts notwendigen Maßnahmen [...] übt der Nationalrat durch einen von ihm gewählten Ausschuß aus [...]“.77 Später interpretierte Seitz im AoKR diesen als „halb eine parlamentarische, halb eine Regierungskörperschaft", weshalb er die Formulierung, wonach einem Entwurf die "Zustimmung erteilt" werde, für unangemessen hielt: Der AoKR habe „Beschluss zu fassen, nicht aber bloss eine Zustimmung zu erteilen". ${ }^{78}$ Das Vollmachtengesetz ließ mit seiner Formulierung, dass ein Beschluss des AoKR dann zustandekam, wenn „die Staatsräte durch einen Beschluß zustimmen",79 aber auch eine andere Interpretation $\mathrm{zu}$.

Tatsächlich wird man für beide Lesarten Argumente finden: Die Staatsräte im AoKR bildeten einen Parlamentsausschuss entsprechend den

\footnotetext{
${ }^{75}$ ANONYMUS, Herr Präsident 1.

${ }^{76}$ BERCHTOLD, Verfassungsgeschichte 360.

77 ANONYMUS, Gesetzgebungsrecht 3.

${ }^{78}$ AoKR Protokoll 34, 3.

${ }^{79}$ Vollmachtengesetz 1922 BGBl. 844/1922, Art. VI.
}

parlamentarischen Kräfteverhältnissen unter Einbindung aller Parteien, sie genossen parlamentarische Immunität - dies spricht für eine Art von Parlamentarismus. Der AoKR wurde durch den Bundeskanzler einberufen, war an der Vorzensur der auf Staatsfinanzen bezogenen Regierungsvorlagen beteiligt und übte seine Funktion als Staatsrat unabhängig von der Gesetzgebungsperiode aus - dies spricht für eine erweiterte Regierung. Es ist daher nicht erstaunlich, dass die Verfassungsrechtslehre den AoKR als einen "Zwitter", eine "halbpathologische Erscheinung des Staatsrechts" oder gar als eine "Absonderlichkeit" bezeichnete, wobei gerade solche Absonderlichkeiten mit dem größten Lehrgehalt einhergehen würden. ${ }^{80}$ Bei Berücksichtigung der Verfassungswirklichkeit wird man den AoKR zwar vielleicht als parlamentarisches Organ qualifizieren können, das jedoch ebenso wie der Nationalrat selbst, und dies bis heute - von der politischen Mehrheit in den Schatten der von dieser Mehrheit getragenen Regierung gestellt wurde. Hinter besonderen Regierungsbeschlüssen als Form außerordentlicher Gesetzgebung stand ebenso wie hinter Nationalratsbeschlüssen zur ordentlichen Gesetzgebung eine politische Mehrheit, welche die Regierung stützte und in der Außenwahrnehmung mit dieser terminologisch verschmolz. Berchtold, der die geringe Begeisterung der politischen Mehrheit für den AoKR darauf zurückführte, dass es sich ,eben um einen verkleinerten Nationalrat“ gehandelt hätte - „Deshalb war nicht allzuviel gewonnen"81 - ist zwar zuzustimmen, jedoch mit der Ergänzung: Auch für die Opposition war im AoKR nichts zu gewinnen.

Unabhängig davon, wie man dieses Gremium nun endgültig einschätzen mag, handelte es sich beim AoKR um ein Instrument der Entdemokra-

\footnotetext{
${ }^{80}$ WITTMAYER, Staatsrecht 391.

${ }^{81}$ BERCHTOLD, Verfassungsgeschichte 361.
} 
tisierung und Entparlamentarisierung. In seiner Kreation manifestiert sich ein erhebliches Misstrauen gegen die Reformfähigkeit eines Staates mit parlamentarischer Demokratie und es erscheint bemerkenswert, dass dieses Misstrauen offenbar auch in Großbritannien, Frankreich, Italien und der Tschechoslowakei anzutreffen war, Ländern, die vielfach als Hort demokratischen Denkens in der sonst demokratiefeindlichen Zwischenkriegszeit stilisiert wurden. Gläubigerinteressen waren offenbar deutlich wichtiger als Vertrauen in die Demokratie Vergleiche mit der Griechenlandkrise des 21. Jahrhunderts drängen sich nicht zufällig auf.

\section{Korrespondenz:}

Prof. Dr. Gerald KoHL

Universität Wien

Institut für Rechts- und Verfassungsgeschichte

Juridicum

Schottenbastei 10-16

A-1010 Wien

gerald.kohl@univie.ac.at

ORCID-Nr. 0000-0002-6812-3897

\section{Abkürzungen:}

AoKR Außerordentlicher Kabinettsrat

GK Generalkommissär

Siehe auch das allgemeine Abkürzungsverzeichnis: [http://www.rechtsgeschichte.at/files/abk.pdf]

\section{Archivalische Quellen}

ÖStA, AdR, MRang, MR 1. Rep, AoKR (2 Kartons: Sitzungsprotokolle 1-22 in Kart. 1, 23-37 in Kart. 2).

\section{Literatur}

Ludwig ADAMOvich, Grundriss des österreichischen Staatsrechtes (Wien ${ }^{2} 1932$ )

Rudolf AgSTNER, Handbuch des Österreichischen Auswärtigen Dienstes, Bd. 1: 1918-1938. Zentrale, Gesandtschaften und Konsulate (Wien 2015).

Hellmut ANDICS, Der Staat, den keiner wollte: Österreich 1918-1938 (Wien ${ }^{21964) . ~}$

ANONYMUS, Der Herr Präsident, in: Reichspost, Nr. 36 v. 7. 2. 1923, 1 .

ANONYMUS, Gesetz, Verordnung und Ermächtigung, in: Arbeiterzeitung, Nr. 1 v. 2. 1. 1923, 1-2.

ANONYMUS, Das Gesetzgebungsrecht und der Kabinettsrat, in: Arbeiterzeitung, Nr.315 v. 23. 11. 1922, 3-4.

ANONYMUS, Seipels neuer Vorschlag, in: Arbeiterzeitung, Nr. 310 v. 18. 11. 1922, 2.

Klaus BERCHTOLD, Verfassungsgeschichte der Republik Österreich, Bd. I: 1918-1933, Fünfzehn Jahre Verfassungskampf (Wien-New York 1998).

Peter BERGER, Kurze Geschichte Österreichs im 20. Jahrhundert (Wien 22008).

Norbert BLIEBERGER, Österreichs Währungskrise und ihre Lösung 1922/23. Genese und Konsequenzen der „Genfer Sanierung“ (Dipl.Arb., WU Wien 1999).

Rudolf FREUnD, Die Genfer Protokolle. Ihre Geschichte und Bedeutung für das Staatsleben DeutschÖsterreichs (= Sozialwissenschaftliche Forschungen V/2, Berlin-Leipzig 1924).

Peter KEUC, Die Haltung der österreichischen Sozialdemokratie zur Sanierung Österreichs, insbesondere zu den Genfer Protokollen von 1922 (Diss. Graz 1968).

Gottlieb LADNER, Seipel als Überwinder der Staatskrise vom Sommer 1922. Zur Geschichte und Entstehung der Genfer Protokolle vom 4. Oktober 1922 (= Publikationen des österreichischen Instituts für Zeitgeschichte 1, Wien-Graz 1964).

Adolf MERKL, Die Völkerbundkontrolle als Staatsrechtsinstitut, in: Zeitschrift für öffentliches Recht $3(1921 / 22)$ 599-627.

Roman SANDGRUBER, Ökonomie und Politik. Österreichische Wirtschaftsgeschichte vom Mittelalter bis zur Gegenwart, hg. v. Herwig WOLfRAM (= Österreichische Geschichte, Wien 2005).

Walter SCHMID, Die innenpolitischen Auseinandersetzungen um die Genfer Protokolle (Hausarb., Univ. Wien 1977).

Christian A. THEINER, Unter Kuratel! Die Genfer Protokolle von $1922 \mathrm{im}$ Spiegel der Tiroler Tagespresse (Dipl.Arb., Univ. Innsbruck 2015). 
Leo WiTTMAYER, Das Staatsrecht des österreichischen Wiederaufbaues, in: Deutsche Juristen-Zeitung 28 (1923) 388-391.

\section{Zeitungen}

Arbeiter-Zeitung, Nr. 310 v. 18. 11. 1922, Nr. 36 v. 7. 2. 1923.

Illustrierte Kronen-Zeitung, Nr. 8281 v. 7. 2. 1923.

Neue Freie Presse, Nr. 20981 v. 7. 2. 1923.

Neues Wiener Tagblatt, Nr. 307 v. 17. 11. 1922, Nr. 345 v. 27. 12. 1922 , Nr. 43 v. 14. 2. 1923.

Reichspost, Nr. 36 v. 7. 2. 1923.

Die Rote Fahne, Nr. 1072 v. 19. 11. 1922.
Salzburger Chronik, Nr. 254 v. 19./20. 11. 1922.

Tages-Post, Nr. 81 v. 10. 4. 1923.

Wiener Zeitung, Nr. 15 v. 19. 1. 1923, Nr. 22 v. 27. 1. 1923, Nr. 30 v. 7. 2. 1923, Nr. 62 v. 16. 3. 1923.

\section{Gesetze}

Genfer Protokolle 1922 BGBl. 842/1922

Wiederaufbaugesetz 1922 BGBl. 843/1922

Vollmachtengesetz 1922 BGBl. 844/1922

(vgl. auch Tabelle 4) 
Abbildung 1: Das System der Genfer Protokolle

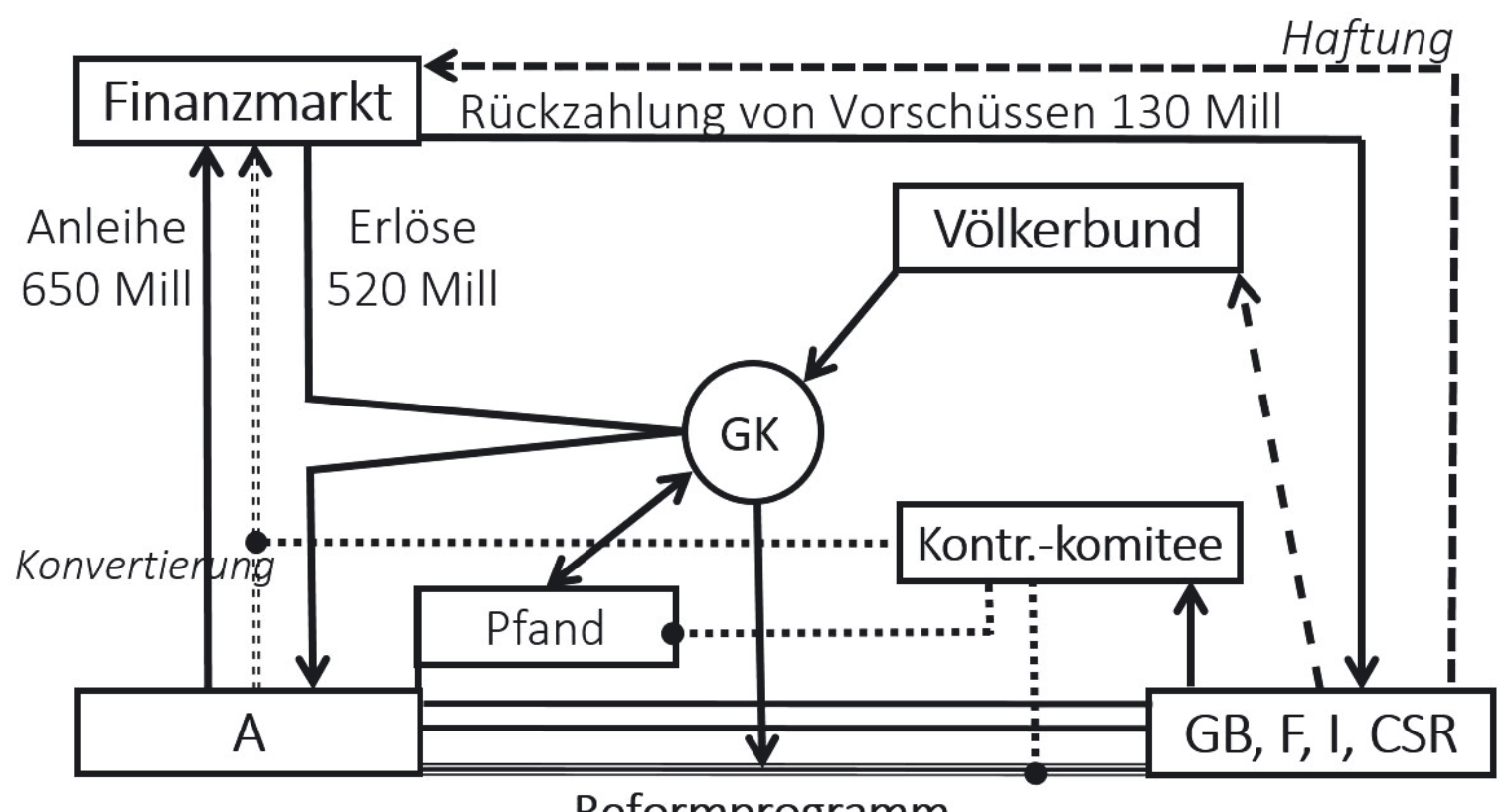

Reformprogramm

Abbildung 2: Beispiel einer Kundmachung im Bundesgesetzblatt

\section{3.}

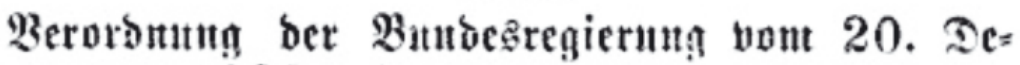
jentber 192\%, betrefients bie Fefitietsung ber Penjionsbeiträge Der Butbes(Butbesverfehr: )= atgefiteflett.

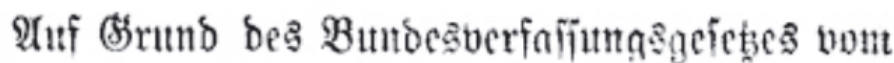
26. November 1922, R. (5), BI. SR. 844, und

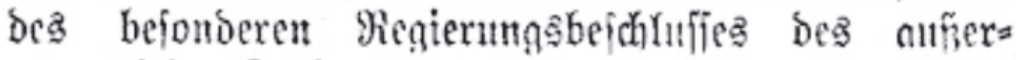
proentyiçen Rabinettîrates boun 20. Dezember 1922 wird ververonet: 
Abbildung 3: Umfang, Dauer und Bedeutung der Sitzungen des AOKR

Sitzungen des außerordentlichen Kabinettsrats 1922-1924

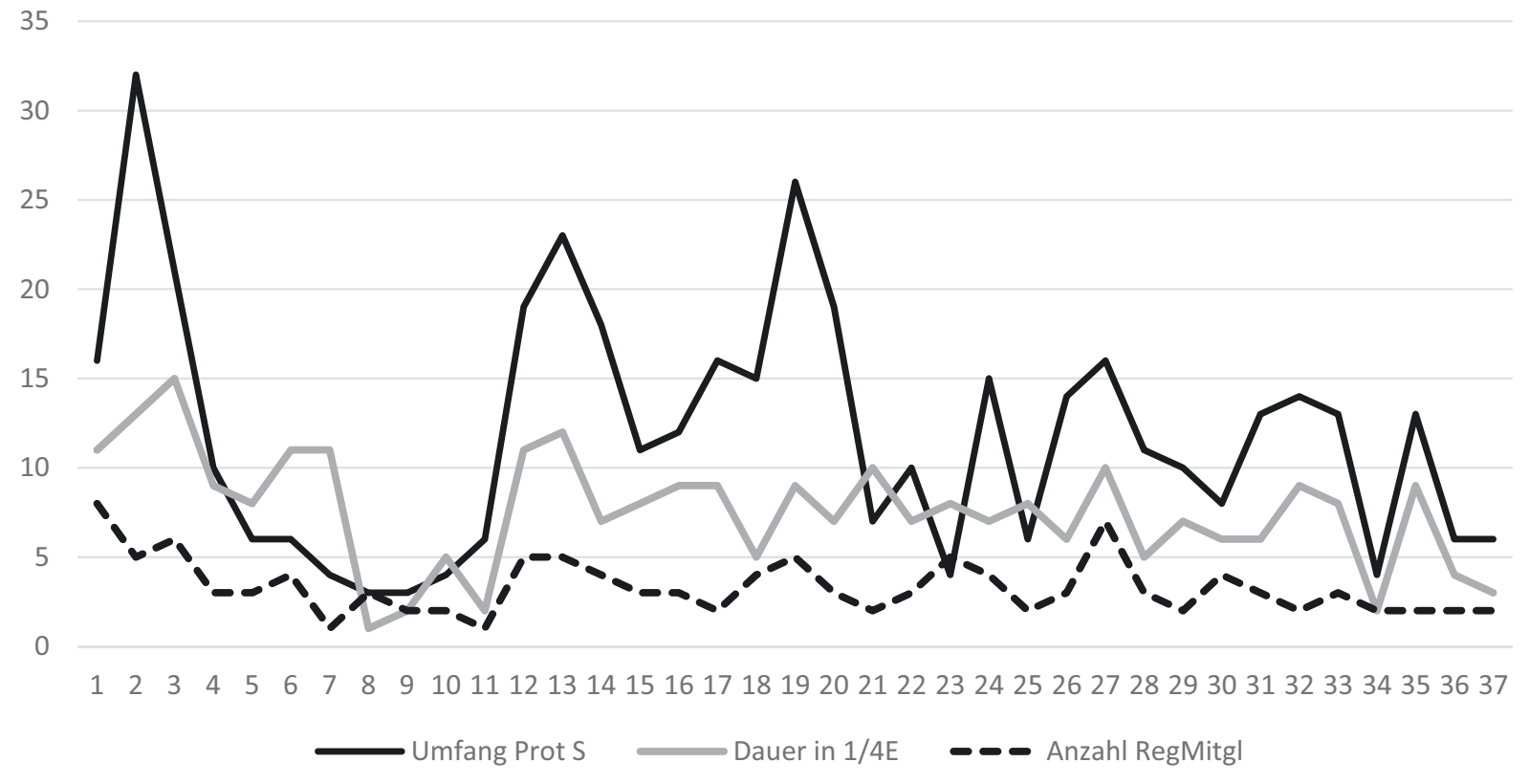

Abbildung 4: Anzahl/Verteilung besonderer Regierungsbeschlüsse (Kundmachung im BGBl)

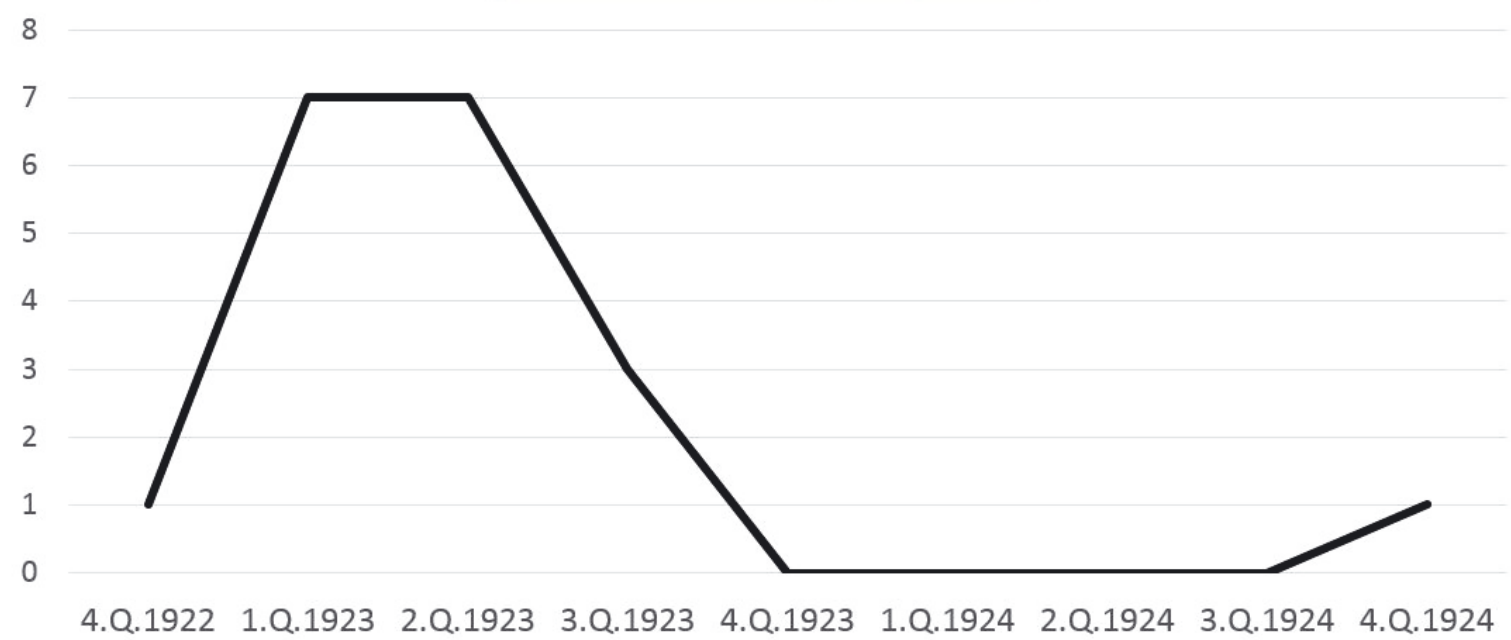


Tabelle 1: Die Staatsräte nach Parteizugehörigkeit und Sitzungsfrequenz

\begin{tabular}{|c|c|c|c|c|}
\hline Name & I. GP & II. GP & Partei & anwesend \\
\hline Schiegl Wilhelm & $\mathrm{H}$ & $\mathrm{H}$ & $\mathrm{SdP}$ & 37 \\
\hline Fink Jodok, Dr. h.c. & $\mathrm{H}$ & $\mathrm{H}$ & CSP & 36 \\
\hline Tomschik Josef & $\mathrm{H}$ & $\mathrm{H}$ & SdP & 36 \\
\hline Partik Matthias & $\mathrm{E}$ & $\mathrm{E}$ & CSP & 34 \\
\hline Bauer Otto, Dr. & $\mathrm{H}$ & $\mathrm{H}$ & SdP & 33 \\
\hline Heinl Eduard, Dr. h.c. & $\mathrm{H}$ & $\mathrm{H}$ & CSP & 33 \\
\hline Steinegger Hans & $\mathrm{H}$ & $\mathrm{E}$ & CSP & 32 \\
\hline Seitz Karl & $\mathrm{H}$ & $\mathrm{H}$ & SdP & 30 \\
\hline Hampel Ernst, Dr. & $\mathrm{H}$ & - & GdP & 29 \\
\hline Waiß $*$ Erwin & $\mathrm{E}$ & E & CSP & 27 \\
\hline Kollmann Josef & $E$ & E & CSP & 26 \\
\hline Schönbauer Ernst, DDr. & $\mathrm{H}$ & - & OK & 26 \\
\hline Schürff Hans, Dr. & $\mathrm{H}$ & - & GdP & 26 \\
\hline Zelenka Franz & $E$ & E & SdP & 25 \\
\hline Heigl Josef & $\mathrm{E}$ & $\mathrm{E}$ & CSP & 24 \\
\hline Dinghofer Franz, Dr. & $\mathrm{H}$ & $\mathrm{H}$ & $\mathrm{GdP}$ & 23 \\
\hline Rudel-Zeynek Olga & $\mathrm{E}$ & $\mathrm{E}$ & CSP & 22 \\
\hline Gürtler Alfred, Dr. & $\mathrm{H}$ & $\mathrm{H}$ & CSP & 21 \\
\hline Hanusch Ferdinand & $\mathrm{H}$ & - & SdP & 21 \\
\hline Wollek Richard & $E$ & - & CSP & 21 \\
\hline Heitzinger Johann & $\mathrm{E}$ & $\mathrm{E}$ & CSP & 20 \\
\hline Eisler Arnold, Dr. & $\mathrm{H}$ & $\mathrm{H}$ & SdP & 18 \\
\hline Sever Albert & $\mathrm{E}$ & $\mathrm{H}$ & SdP & 18 \\
\hline Geisler Simon & $\mathrm{E}$ & $\mathrm{E}$ & CSP & 17 \\
\hline Leuthner Karl & $\mathrm{E}$ & $\mathrm{E}$ & SdP & 17 \\
\hline Danneberg Robert, Dr. & $\mathrm{H}$ & $\mathrm{H}$ & SdP & 14 \\
\hline Resch Josef, Dr. & $E$ & - & CSP & 14 \\
\hline Ellenbogen Wilhelm, Dr. & $\mathrm{H}$ & $\mathrm{H}$ & SdP & 13 \\
\hline Schulz Hermann & $\mathrm{E}$ & $\mathrm{E}$ & SdP & 13 \\
\hline Weiskirchner Richard, Dr. & $\mathrm{H}$ & - & CSP & 13 \\
\hline Eldersch Matthias & $\mathrm{H}$ & $\mathrm{H}$ & SdP & 12 \\
\hline Födermayr Florian & $\mathrm{H}$ & $\mathrm{H}$ & CSP & 12 \\
\hline Renner Karl, Dr. & $\mathrm{H}$ & $\mathrm{H}$ & SdP & 12 \\
\hline Deutsch Julius, Dr. & $\mathrm{E}$ & $\mathrm{E}$ & SdP & 11 \\
\hline Kletzmayr Hermann & $E$ & $E$ & CSP & 10 \\
\hline Volker Otto & $\mathrm{E}$ & $\mathrm{E}$ & CSP & 10 \\
\hline Glöckel Otto & $\mathrm{E}$ & E & SdP & 9 \\
\hline Pauly Max & $\mathrm{E}$ & - & $\mathrm{GdP}$ & 9 \\
\hline Gimpl Georg, Dr. & $E$ & - & CSP & 8 \\
\hline Freundlich Emmy & $\mathrm{E}$ & $\mathrm{E}$ & $\mathrm{SdP}$ & 7 \\
\hline Angerer Hans, Dr. & $E$ & E & $\mathrm{GdP}$ & 5 \\
\hline Gürtler Johann & $\mathrm{E}$ & $\mathrm{E}$ & CSP & 5 \\
\hline Zwetzbacher Josef & $\mathrm{H}$ & $\mathrm{H}$ & CSP & 5 \\
\hline
\end{tabular}




\begin{tabular}{|l|c|c|c|c|}
\hline Eisenhut Josef & E & E & CSP & 4 \\
\hline Pirchegger Anton & E & H & CSP & 4 \\
\hline Krüzner ** Alexander, Dr. & E & - & CSP & 3 \\
\hline Miklas Wilhelm & - & H & CSP & 3 \\
\hline Rehrl Franz, Dr. & H & H & CSP & 3 \\
\hline Schumacher Franz, Dr. & - & H & CSP & 3 \\
\hline Spalowsky Franz & - & H & CSP & 3 \\
\hline Stempfer Georg & E & - & CSP & 3 \\
\hline Stradal Emmy & E & - & GdP & 3 \\
\hline Hauser Johann Nepomuk & E & H & CSP & 2 \\
\hline Hueber Anton & E & H & SdP & 2 \\
\hline Paulitsch Michael & H & H & CSP & 2 \\
\hline Richter Paul & - & E & SdP & 2 \\
\hline Rintelen Anton, Dr. & H & - & CSP & 2 \\
\hline Sailer Georg & E & E & SdP & 2 \\
\hline Waber Leopold, Dr. & - & E & GdP & 2 \\
\hline Allina Heinrich & E & E & SdP & 1 \\
\hline Austerlitz Friedrich & E & E & SdP & 1 \\
\hline Forstner August & E & E & SdP & 1 \\
\hline Größbauer Philipp & E & - & OK & 1 \\
\hline Reiner Fritz, Ing. & - & E & CSP & 1 \\
\hline Scheibein Wilhelm & - & E & SdP & 1 \\
\hline Schwinner Josef, Dr. & E & E & CSP & 1 \\
\hline Stöckler Josef & E & E & CSP & CSP \\
\hline Sturm Josef & & - & GdP & 1 \\
\hline Ursin Josef, Dr. & & & 1 \\
\hline
\end{tabular}

* laut Protokollen stets „Dr. Waiß“, in den Protokollen zur 6. und 34. Sitzung „Dr. Waiss"

** laut Protokollen stets „Dr. Krützner“

Abkürzungen:

CSP Christlichsoziale Partei

E Ersatzmitglied des AoKR

GdP Großdeutsche Volkspartei

$\mathrm{H} \quad$ Hauptmitglied des AoKR

OK ohne Klubzugehörigkeit

SdP Sozialdemokratische Partei 


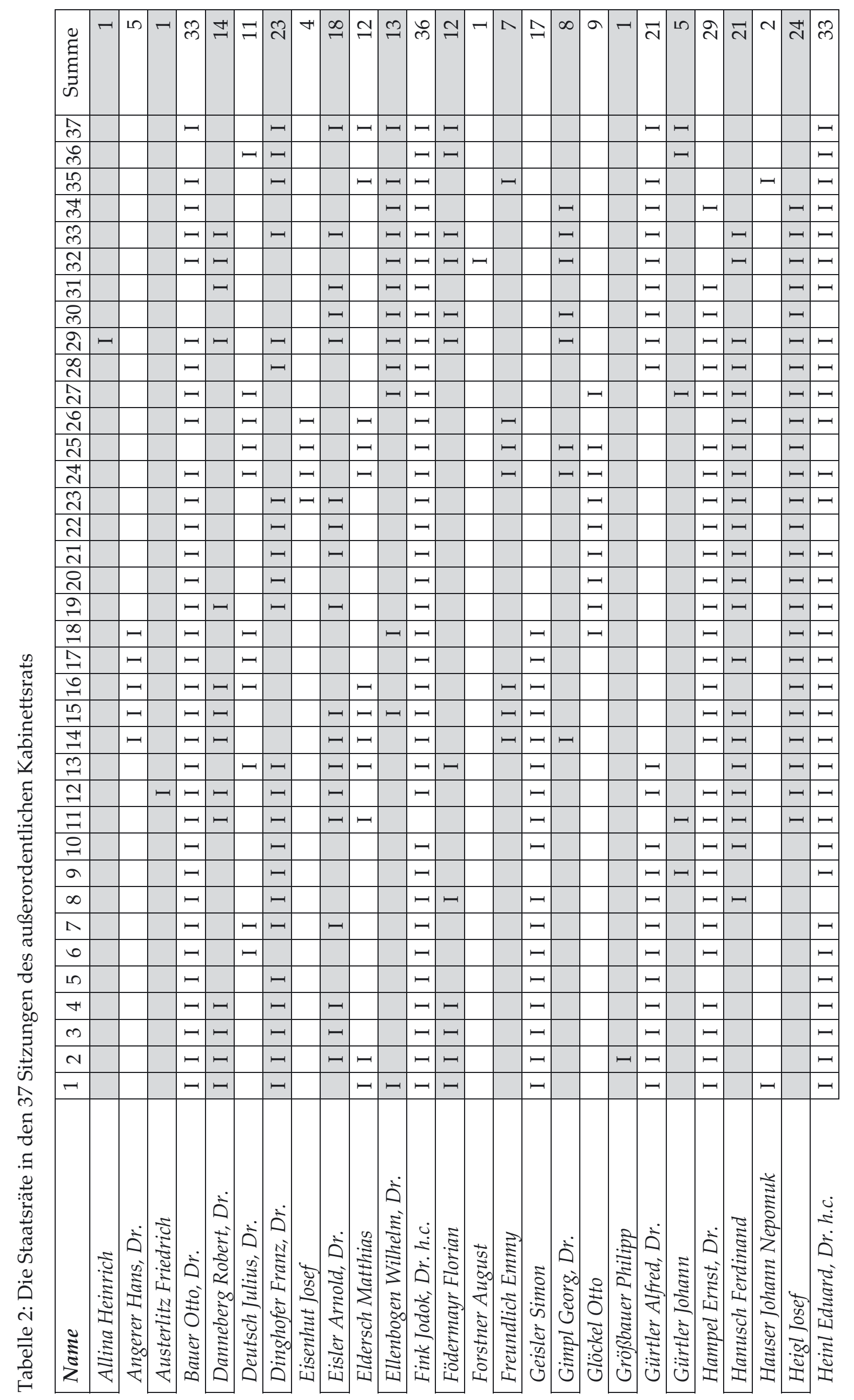




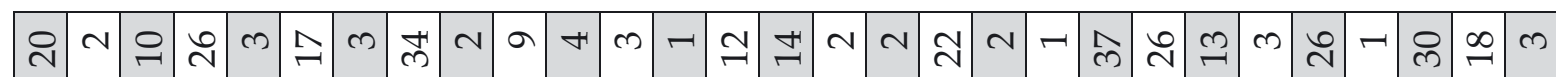

\begin{tabular}{|c|c|c|c|c|c|c|c|c|c|c|c|c|c|c|c|c|c|c|c|c|c|c|}
\hline & $\pi$ & - & & - & -1 & & & & & 7 & & I & & $\varpi$ & & -1 & & -7 & & & & -1 \\
\hline & & - & & $\neg$ & $\neg 1$ & 4 & & - & & & -1 & $1-$ & & $\pi$ & $-1-$ & -1 & & - & & & & -1 \\
\hline & -1 & - & & & -7 & 41 & & • & & & & & & & & -1 & & 1 & & -1 & - & -1 \\
\hline-1 & & & & & 7 & & & & & & 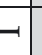 & & & & & -1 & & & & $7-$ & 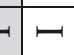 & \\
\hline- & & & & & & & t & & & & - & & & & & - & -1 & & & & & \\
\hline- & & & & $\varpi$ & & & - & & & & & & & & - & -1 & & & & & $\square$ & \\
\hline- & & & & & & & - & & -1 & & & & & 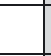 & & -1 & -1 & & & & $\pi$ & \\
\hline - & & & & . & & & - & & -1 & & & & & - & & $\neg$ & - & & 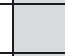 & 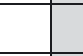 & & \\
\hline-1 & & & & -1 & & & $\tau$ & & - & & & & & - & & - & - & & & - & & \\
\hline 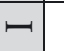 & & & 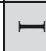 & - & & & & & & & & & & -1 & & $\neg$ & - & & -1 & 1 & & \\
\hline- & & & - & -1 & & & 1 & & & & & & & -1 & & -1 & - & & -1 & $1-$ & & \\
\hline - & & - & & . & & & - & & & & & & & -1 & & 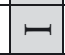 & & & - & -7 & & \\
\hline- & & - & - & & & & 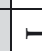 & & & & & & & 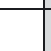 & & $\neg$ & - & & - & & $\varpi$ & \\
\hline - & & 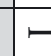 & & $\varpi$ & & & -1 & & & & -1 & & & 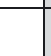 & & $\neg$ & & & - & $1-$ & & \\
\hline - & & - & & -1 & & & & & & & -1 & & & -1 & & - & -1 & & - & - & & \\
\hline-1 & & 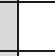 & & - & & & & & & & & & & - & & - & & & - & - & & \\
\hline- & & 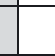 & & $\square$ & & & & & & & & & & - & & 7 & 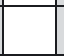 & & -1 & -1 & & \\
\hline- & & - & & 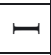 & & & & & & & -1 & & & $\varpi$ & & 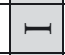 & - & & - & - & & \\
\hline 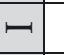 & & - & & 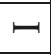 & & & & & & & & & & -1 & & - & - & & - & - & & \\
\hline-1 & & - & & - & & & & & & & & 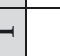 & & - & & - & - & & - & - & 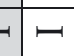 & \\
\hline- & & - & & 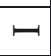 & & & & & & & & & & $\square$ & & - & - & & -1 & & $\pi$ & \\
\hline- & & - & & 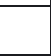 & & & & 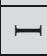 & & & & & & 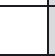 & & - & - & & - & & $\pi$ & \\
\hline- & & - & & & & & & $\varpi$ & & & & -1 & & 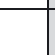 & & $\neg$ & $\neg$ & & 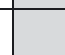 & -1 & 4 & \\
\hline & & - & & 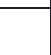 & & & & & & & 7 & & - & . & & $\neg$ & -1 & & $\square$ & - & & \\
\hline & & - & & 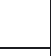 & & & & & & & & & & 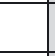 & & - & -1 & - & - & - & 4 & \\
\hline & & - & & 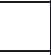 & & & & & & & & & & 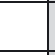 & & - & & - & - & - & & \\
\hline & & - & & & & & & & & & & & & 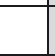 & & - & -1 & & -1 & -1 & 4 & \\
\hline & & - & & . & & & & & & & & & & 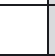 & & -1 & -1 & -1 & - & & $\pi$ & \\
\hline & & $4-$ & & 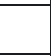 & & & & & & & & & & - & & -1 & -1 & - & 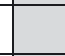 & & 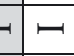 & \\
\hline & & 47 & & - & & & & & & & & & & -1 & & $\neg$ & -1 & $\neg$ & -1 & & - & \\
\hline & & $41-$ & & 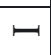 & & & & & & & & & & - & & $\square$ & -1 & -1 & -1 & -1 & $\pi$ & \\
\hline & & 4 & & 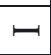 & & & & & & & & & & - & & 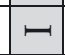 & -1 & -1 & -1 & $1-$ & $\pi$ & \\
\hline & & 41 & & & & & & & & & -1 & & & -1 & & - & & - & - & & $\pi$ & \\
\hline & & 41 & & & & & & & & & 7 & & & - & & - & -1 & -7 & - & - & $\pi$ & \\
\hline & & $4-$ & & & & & & & & & -1 & & & - & & - & -1 & - & - & $1-$ & -1 & \\
\hline & & 4 & & & & & & & & & - & & & - & & - & - & - & - & - & 4 & \\
\hline & & & & & 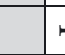 & & & & & & -1 & & ロ & & & 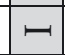 & -1 & -1 & 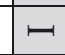 & 1 & 4 & \\
\hline
\end{tabular}

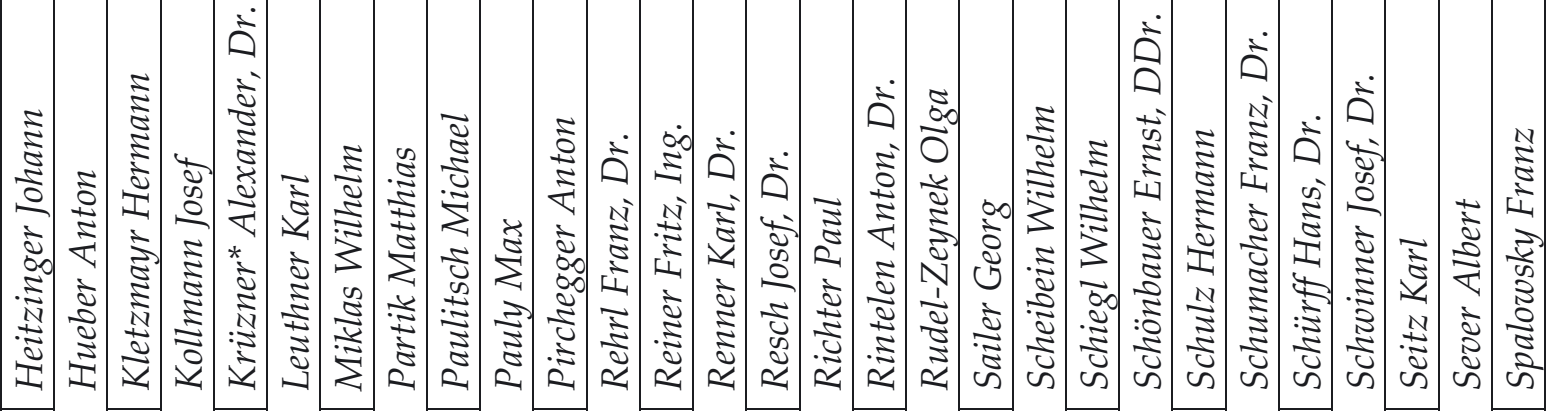




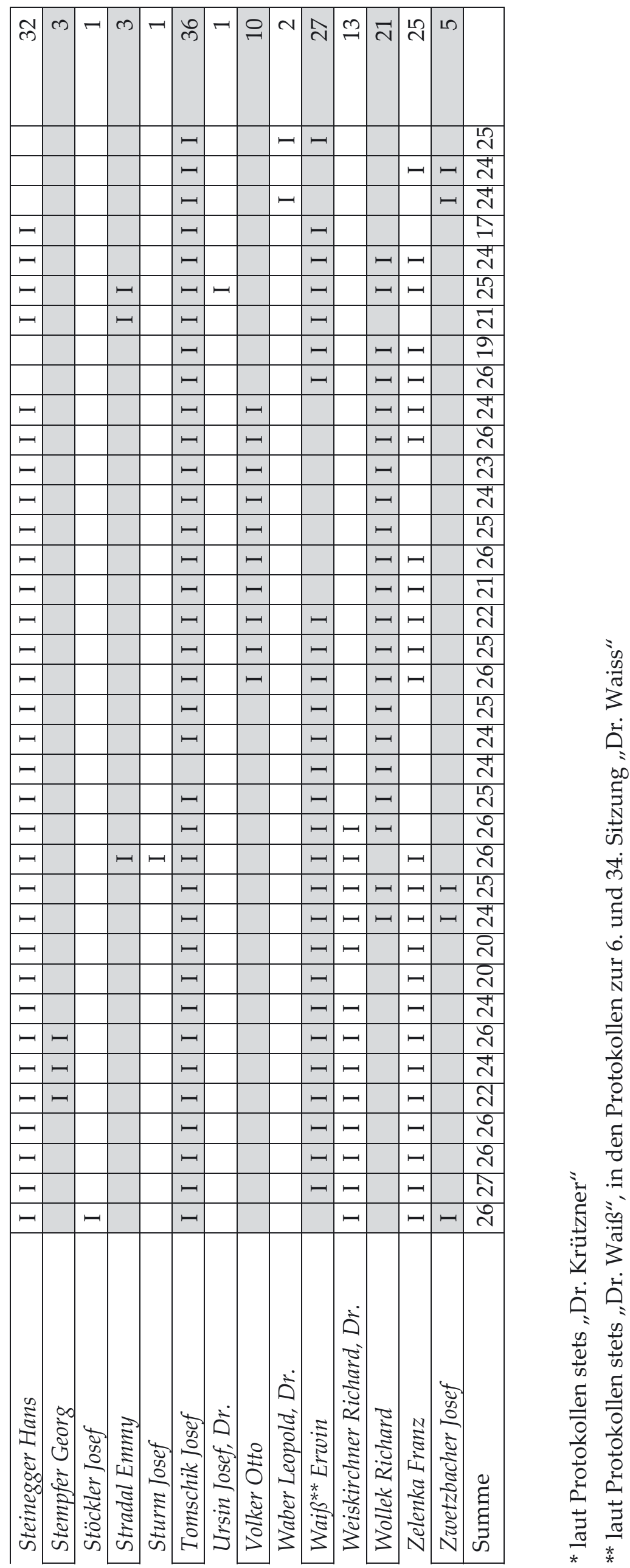




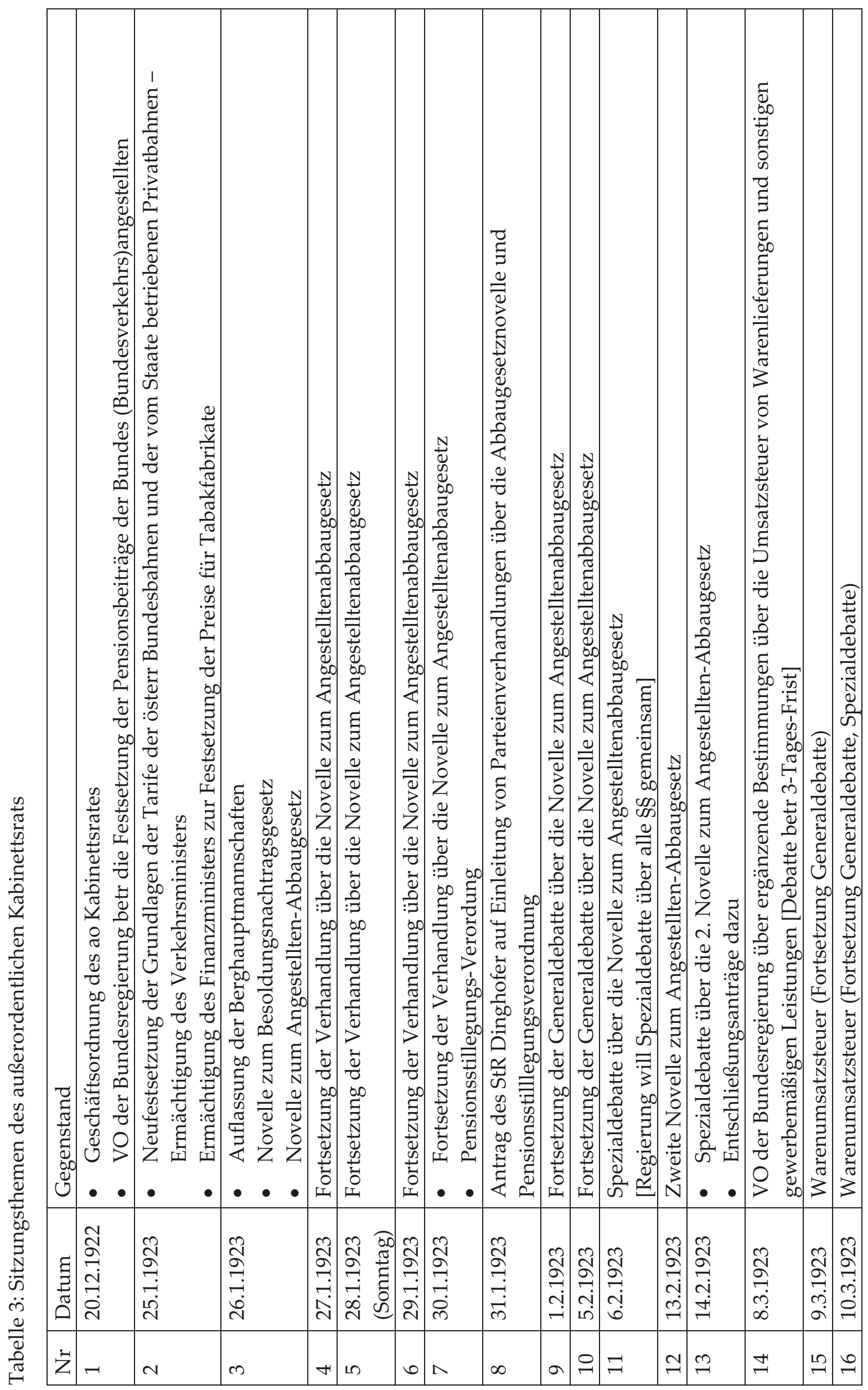




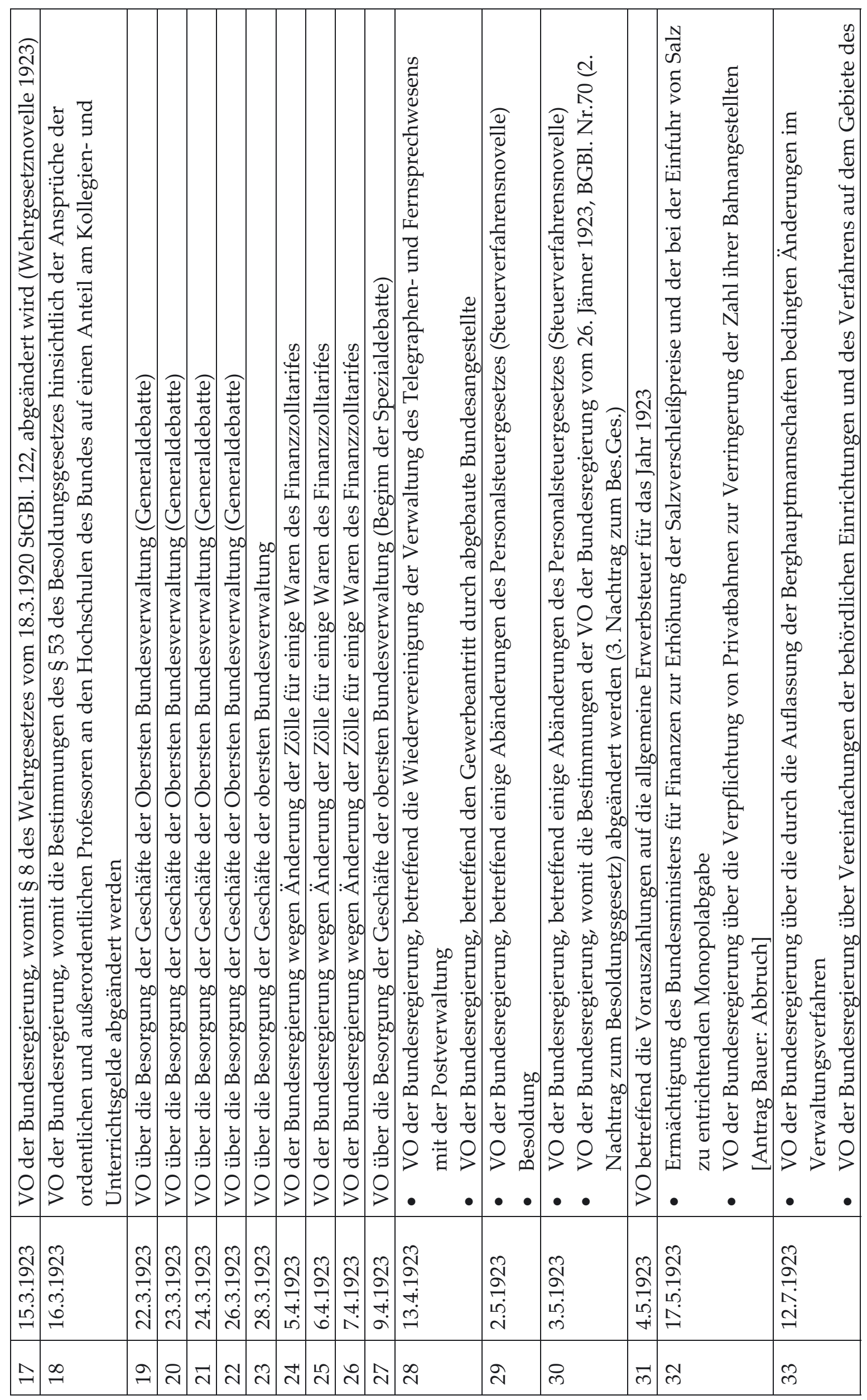




\begin{tabular}{|c|c|c|c|c|}
\hline 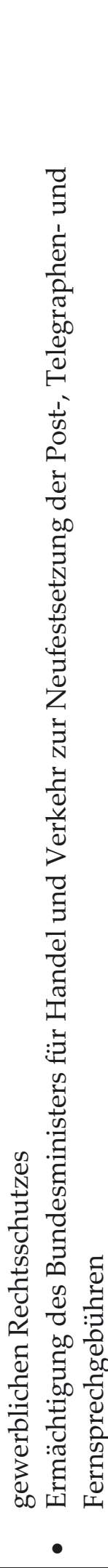 & 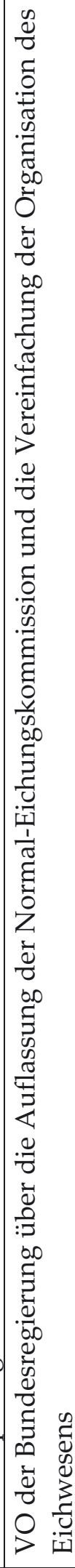 & 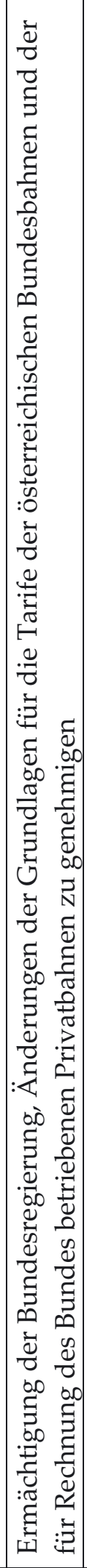 & 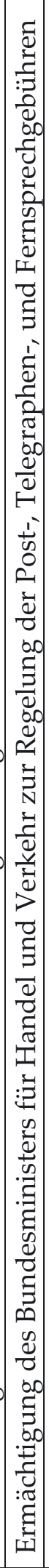 & 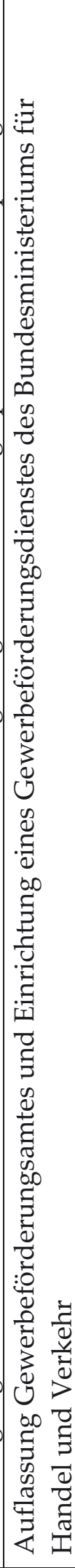 \\
\hline & 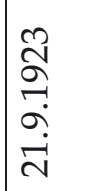 & 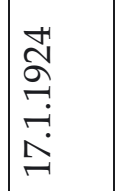 & 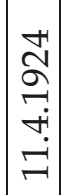 & 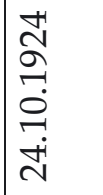 \\
\hline & கு & $\stackrel{1}{m}$ & లి & ले \\
\hline
\end{tabular}


Tabelle 4: Das legislative Werk des AoKR

\begin{tabular}{|c|c|}
\hline $\begin{array}{l}\text { Verordnung der Bundesregierung vom 20. Dezember 1922, } \\
\text { betreffend die Festsetzung der Pensionsbeiträge der } \\
\text { Bundes(Bundesverkehrs)angestellten }\end{array}$ & BGB1. 935/1922 \\
\hline $\begin{array}{l}\text { Verordnung der Bundesregierung vom 26. Jänner } 1923 \text { über die } \\
\text { Auflassung der Berghauptmannschaften }\end{array}$ & BGB1. 69/1923 \\
\hline $\begin{array}{l}\text { Verordnung der Bundesregierung vom 26. Jänner 1923, womit einige } \\
\text { Bestimmungen des Bundesgesetzes vom 28. Juni 1922, B. G. Bl. Nr. } \\
\text { 367, betreffend die Bezüge der Bundesangestellten } \\
\text { (Besoldungsnachtragsgesetz), abgeändert werden (2. Nachtrag zum } \\
\text { Besoldungsgesetz) }\end{array}$ & BGBl. 70/1923 \\
\hline $\begin{array}{l}\text { Verordnung der Bundesregierung vom 9. Februar 1923, womit die } \\
\text { Bestimmungen des Bundesgesetzes vom 24. Juli 1922, B. G. Bl. Nr. } \\
\text { 499, betreffend Maßnahmen zur Verringerung der Zahl der } \\
\text { Bundes(Bundesverkehrs)angestellten (Angestellten-Abbaugesetz), } \\
\text { abgeändert und ergänzt werden (Angestellten-Abbaugesetznovelle) }\end{array}$ & BGB1. 82/1923 \\
\hline $\begin{array}{l}\text { Verordnung der Bundesregierung vom 14. Februar 1923, womit die } \\
\text { Bestimmungen des Bundesgesetzes vom 24. Juli 1922, B. G. Bl. Nr. } \\
\text { 499, betreffend Maßnahmen zur Verringerung der Zahl der } \\
\text { Bundes(Bundesverkehrs)angestellten (Angestellten-Abbaugesetz), } \\
\text { abgeändert und ergänzt werden (2. Angestellten- } \\
\text { Abbaugesetznovelle) }\end{array}$ & BGB1. 91/1923 \\
\hline $\begin{array}{l}\text { Verordnung der Bundesregierung vom 10. März 1923, betreffend } \\
\text { ergänzende Bestimmungen über die Umsatzsteuer von } \\
\text { Warenlieferungen und sonstigen gewerbsmäßigen Leistungen } \\
\text { (Warenumsatzsteuer) }\end{array}$ & BGB1. 120/1923 \\
\hline $\begin{array}{l}\text { Verordnung der Bundesregierung vom 15. März } 1923 \text { womit } \S 8 \text { des } \\
\text { Wehrgesetzes vom 18. März 1920, BGB1. Nr. 122, abgeändert wird } \\
\text { (Wehrgesetznovelle vom Jahre 1923) }\end{array}$ & BGBl. 145/1923 \\
\hline $\begin{array}{l}\text { Verordnung der Bundesregierung vom 17. März 1923, womit die } \\
\text { Bestimmungen des § } 53 \text { des Bundesgesetzes vom 13. Juli 1921, B. G. } \\
\text { Bl. Nr. 376, zur Regelung der Besoldungsverhältnisse der } \\
\text { Bundesangestellten (Besoldungsgesetz), hinsichtlich des Anspruches } \\
\text { der ordentlichen und außerordentlichen Professoren an den } \\
\text { Hochschulen des Bundes auf einen Anteil am Kollegien- und } \\
\text { Unterrichtsgelde abgeändert werden }\end{array}$ & BGBl. 147/1923 \\
\hline $\begin{array}{l}\text { Verordnung der Bundesregierung vom 7. April } 1923 \text { wegen } \\
\text { Änderung der Zölle für einige Waren des Finanzzolltarifes }\end{array}$ & BGBl. 189/1923 \\
\hline $\begin{array}{l}\text { Verordnung der Bundesregierung vom 9. April } 1923 \text { über die } \\
\text { Besorgung der Geschäfte der obersten Bundesverwaltung }\end{array}$ & BGBl. 199/1923 \\
\hline $\begin{array}{l}\text { Verordnung der Bundesregierung vom 13. April 1923, betreffend die } \\
\text { Wiedervereinigung der Verwaltung des Telegraphen- und } \\
\text { Fernsprechwesens mit der Postverwaltung }\end{array}$ & BGB1. 210/1923 \\
\hline Verordnung der Bundesregierung vom 13. April 1923, betreffend den & BGB1. 217/1923 \\
\hline
\end{tabular}




\begin{tabular}{|c|c|}
\hline Gewerbeantritt durch abgebaute Bundesangestellte & \\
\hline $\begin{array}{l}\text { Verordnung der Bundesregierung vom 3. Mai 1923, betreffend einige } \\
\text { Abänderungen des Personalsteuergesetzes (Steuerverfahrensnovelle) }\end{array}$ & BGB1. 243/1923 \\
\hline $\begin{array}{l}\text { Verordnung der Bundesregierung vom 4. Mai 1923, betreffend die } \\
\text { Vorauszahlungen auf die allgemeine Erwerbsteuer für das Jahr } 1923\end{array}$ & BGB1. 244/1923 \\
\hline $\begin{array}{l}\text { Verordnung der Bundesregierung vom 3. Mai 1923, womit die } \\
\text { Bestimmungen der Verordnung der Bundesregierung vom 26. Jänner } \\
\text { 1923, B. G. Bl. Nr. } 70 \text { (2. Nachtrag zum Besoldungsgesetz), } \\
\text { abgeändert werden (3. Nachtrag zum Besoldungsgesetz) }\end{array}$ & BGB1. 257/1923 \\
\hline $\begin{array}{l}\text { Verordnung der Bundesregierung vom 12. Juli } 1923 \text { über die durch } \\
\text { die Auflassung der Berghauptmannschaften bedingten Änderungen } \\
\text { im Verwaltungsverfahren }\end{array}$ & BGB1. 388/1923 \\
\hline $\begin{array}{l}\text { Verordnung der Bundesregierung vom 12. Juli } 1923 \text { über } \\
\text { Vereinfachungen der behördlichen Einrichtungen und des } \\
\text { Verfahrens auf dem Gebiete des gewerblichen Rechtsschutzes }\end{array}$ & BGBl. 392/1923 \\
\hline $\begin{array}{l}\text { Verordnung der Bundesregierung vom 21. September } 1923 \text { über die } \\
\text { Auflassung der Normal-Eichungs-Kommission und die } \\
\text { Vereinfachung der Organisation des Eichwesens }\end{array}$ & BGB1. 550/1923 \\
\hline $\begin{array}{l}\text { Verordnung der Bundesregierung vom } 24 \text {. Oktober } 1924 \text { über die } \\
\text { Auflassung des Gewerbeförderungsamtes und die Einrichtung eines } \\
\text { Gewerbeförderungsdienstes des Bundesministeriums für Handel } \\
\text { und Verkehr }\end{array}$ & BGB1. 409/1924 \\
\hline
\end{tabular}

